\title{
ISOGEOMETRIC ANALYSIS FOR SINGULARLY PERTURBED PROBLEMS IN 1-D: ERROR ESTIMATES*
}

\author{
CHRISTOS XENOPHONTOS ${ }^{\dagger}$ AND IRENE SYKOPETRITOU ${ }^{\dagger}$
}

\begin{abstract}
We consider one-dimensional singularly perturbed boundary value problems of reaction-convectiondiffusion type, and the approximation of their solution using isogeometric analysis. In particular, we use a Galerkin formulation with B-splines as basis functions, defined on appropriately chosen knot vectors. We prove robust exponential convergence in the energy norm, independently of the singular perturbation parameters, and illustrate our findings through a numerical example.
\end{abstract}

Key words. singularly perturbed problem, reaction-convection-diffusion, boundary layers, isogeometric analysis, robust exponential convergence

\section{AMS subject classifications. $65 \mathrm{~N} 30$}

1. Introduction. We consider second order singularly perturbed problems (SPPs) in onedimension, of reaction-convection-diffusion type, whose solution contains boundary layers; see, e.g., [13]. The approximation of the solution to SPPs has received a lot of attention in the last few decades, mainly using finite differences (FDs) and finite elements (FEs) on layer adapted meshes; see, e.g., [9]. Various formulations and results are available in the literature, both theoretical and computational (cf. [9, 13], and the references therein). One method that has not, to our knowledge, been applied to general SPPs is isogeometric analysis (IGA). Since the introduction of IGA by T. R. Hughes et al. [7], the method has been successfully applied to a large number of problem classes. Even though much attention has been given to convection-dominated problems [3], the method has not been applied, as far as we know, to a typical singularly perturbed problem, such as (2.1), (2.2) ahead.

Our goal in this article is to provide the theoretical justification for the application of IGA to SPPs and, in particular, for the approximation of the solution to the boundary value problem (BVP) given by (2.1), (2.2). We use a Galerkin formulation with B-splines as basis functions and select appropriate knot vectors, such that as the polynomial degree increases, the error in the approximation, measured in the energy norm, decays exponentially and independently of the singular perturbation parameters. This is the analog of performing $p$ refinement in the FEM. Even though we focus on the one-dimensional case, our results will serve as the stepping stone to higher dimensions for two reasons: the boundary layer effect is one dimensional (in the direction normal to the boundary) and B-splines in two-dimensions are constructed via tensor products. Hence, this is the first step towards higher dimensions.

The rest of the paper is organized as follows: in Section 2 we describe the model problem, its regularity and the Galerkin formulation we will use. In Section 3 we give a brief introduction to IGA, as described in [3], and present the discrete problem. Section 4 contains our main result of uniform exponential convergence and finally, Section 5 shows the result of a numerical experiment to illustrate the theory.

With $I \subset \mathbb{R}$ an interval with boundary $\partial I$ and measure $|I|$, we will denote by $C^{k}(I)$ the space of continuous functions on $I$ with continuous derivatives up to order $k$. We will use the usual Sobolev spaces $W^{k, m}(I)$ of functions on $I$ with $0,1,2, \ldots, k$ generalized derivatives in $L^{m}(I)$, equipped with the norm and seminorm $\|\cdot\|_{k, m, I}$ and $|\cdot|_{k, m, I}$, respectively. When

* Received October 2, 2018. Accepted November 8, 2019. Published online on January 16, 2020. Recommended by Torsten $\operatorname{Lin} \beta$.

${ }^{\dagger}$ Department of Mathematics and Statistics, University of Cyprus, P.O. Box 20537, 1678 Nicosia, Cyprus (xenophontos@ucy.ac.cy). 
$m=2$, we will write $H^{k}(I)$ instead of $W^{k, 2}(I)$, and for the norm and seminorm, we will write $\|\cdot\|_{k, I}$ and $|\cdot|_{k, I}$, respectively. The usual $L^{2}(I)$ inner product will be denoted by $\langle\cdot, \cdot\rangle_{I}$, with the subscript omitted when there is no confusion. We will also use the space

$$
H_{0}^{1}(I)=\left\{u \in H^{1}(I):\left.u\right|_{\partial I}=0\right\} .
$$

The norm of the space $L^{\infty}(I)$ of essentially bounded functions is denoted by $\|\cdot\|_{\infty, I}$. Finally, the letter $C$ will denote a generic positive constant, independent of any parameters and possibly having different values in each occurence.

2. The model problem and its regularity. We consider the following model BVP: find $u$ such that

$$
\begin{aligned}
-\varepsilon_{1} u^{\prime \prime}(x)+\varepsilon_{2} b(x) u^{\prime}(x)+c(x) u(x) & =f(x), \quad x \in I=(0,1), \\
u(0)=u(1) & =0
\end{aligned}
$$

where $0<\varepsilon_{1}, \varepsilon_{2} \leq 1$ are given parameters that can approach zero and the functions $b, c, f$ are given and sufficiently smooth. We assume that there exist constants $\beta, \gamma, \rho$, independent of $\varepsilon_{1}, \varepsilon_{2}$, such that $\forall x \in \bar{I}$,

$$
b(x) \geq \beta \geq 0, c(x) \geq \gamma>0, c(x)-\frac{\varepsilon_{2}}{2} b^{\prime}(x) \geq \rho>0 .
$$

The reason for the above assumptions is to ensure existence and uniqueness of a weak solution to $(2.1),(2.2)$.

The structure of the solution to (2.1) depends on the roots of the characteristic equation associated with the differential operator. For this reason, we let $\lambda_{0}(x), \lambda_{1}(x)$ be the solutions of the characteristic equation and set

$$
\mu_{0}=-\max _{x \in[0,1]} \lambda_{0}(x), \mu_{1}=\min _{x \in[0,1]} \lambda_{1}(x),
$$

or equivalently,

$$
\mu_{0,1}=\min _{x \in[0,1]} \frac{\mp \varepsilon_{2} b(x)+\sqrt{\varepsilon_{2}^{2} b^{2}(x)+4 \varepsilon_{1} c(x)}}{2 \varepsilon_{1}} .
$$

The following holds true [14, 17]:

$$
\left\{\begin{array}{c}
1 \ll \mu_{0} \leq \mu_{1}, \quad \frac{\varepsilon_{2}}{\varepsilon_{2}+\varepsilon_{1}^{1 / 2}} \leq C \varepsilon_{2} \mu_{0} \leq C, \quad \varepsilon_{1}^{1 / 2} \mu_{0} \leq C, \\
\max \left\{\mu_{0}^{-1}, \varepsilon_{1} \mu_{1}\right\} \leq C \varepsilon_{1}+\varepsilon_{2}^{1 / 2}, \quad \varepsilon_{2} \leq C \varepsilon_{1} \mu_{1}, \\
\text { for } \varepsilon_{2}^{2} \geq \varepsilon_{1}: \varepsilon_{1}^{-1 / 2} \leq C \mu_{1} \leq C \varepsilon_{1}^{-1} \\
\text { for } \varepsilon_{2}^{2} \leq \varepsilon_{1}: \varepsilon_{1}^{-1 / 2} \leq C \mu_{1} \leq C \varepsilon_{1}^{-1 / 2}
\end{array}\right.
$$

The values of $\mu_{0}, \mu_{1}$ determine the strength of the boundary layers and since $\left|\lambda_{0}(x)\right|<\left|\lambda_{1}(x)\right|$ the layer at $x=1$ is stronger than the layer at $x=0$. Essentially, there are three regimes, as shown in Table 1, which is taken from [9]. Figure 2.1 shows the behavior of the solution to (2.1), (2.2), in all three regimes.

The above considerations suggest the following two cases:

1. $\varepsilon_{1}$ is large compared to $\varepsilon_{2}$ : this is similar to a 'regular perturbation' of reactiondiffusion type. If we consider the limiting case $\varepsilon_{2}=0$, then we see that there are two boundary layers, one at each endpoint, of width $O\left(\varepsilon_{1}^{1 / 2}\left|\ln \varepsilon_{1}^{1 / 2}\right|\right)$. This situation has been studied in the literature (see, e.g., [10]) and will not be considered further in this article. 
TABLE 2.1

Different regimes based on the relationship between $\varepsilon_{1}$ and $\varepsilon_{2}$.

\begin{tabular}{cccc} 
& & $\mu_{0}$ & $\mu_{1}$ \\
\hline convection-diffusion & $\varepsilon_{1} \ll \varepsilon_{2}=1$ & 1 & $\varepsilon_{1}^{-1}$ \\
convection-reaction-diffusion & $\varepsilon_{1} \ll \varepsilon_{2}^{2} \ll 1$ & $\varepsilon_{2}^{-1}$ & $\varepsilon_{2} / \varepsilon_{1}$ \\
reaction-diffusion & $\varepsilon_{2}^{2} \ll \varepsilon_{1} \ll 1$ & $\varepsilon_{1}^{-1 / 2}$ & $\varepsilon_{1}^{-1 / 2}$
\end{tabular}

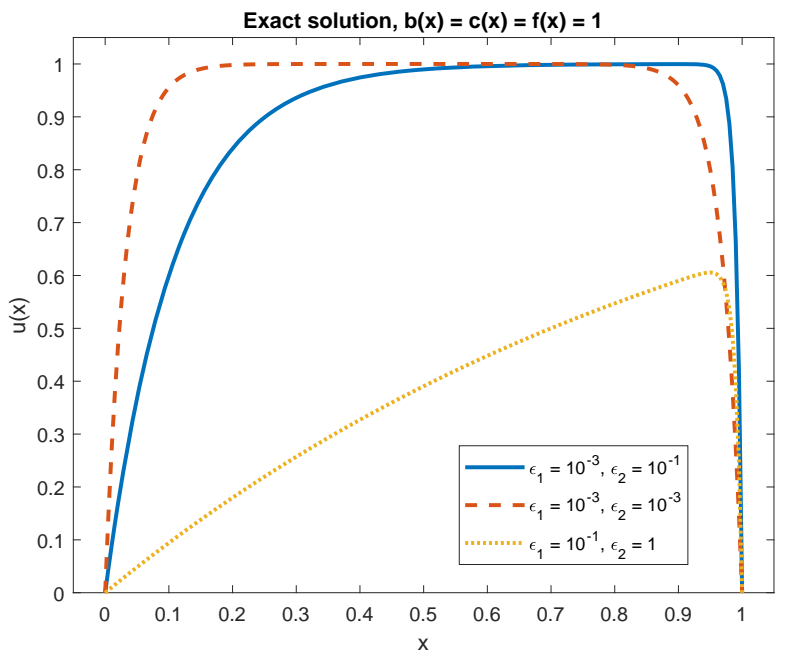

FIG. 2.1. Exact solution for different values of $\varepsilon_{1}$ and $\varepsilon_{2}$.

2. $\varepsilon_{1}$ is small compared to $\varepsilon_{2}$ : before discussing the different regimes, it is instructive to consider the limiting case $\varepsilon_{1}=0$. Then there is an exponential layer (of length scale $O\left(\varepsilon_{2}\right)$ ) at the left endpoint. The homogeneous equation suggests that the different regimes are $\varepsilon_{1} \ll \varepsilon_{2}^{2}, \varepsilon_{1} \approx \varepsilon_{2}^{2}, \varepsilon_{1} \gg \varepsilon_{2}^{2}$.

(a) In the regime $\varepsilon_{1} \ll \varepsilon_{2}^{2}$, we have $\mu_{0}=O\left(\varepsilon_{2}^{-1}\right)$ and $\mu_{1}=O\left(\varepsilon_{2} \varepsilon_{1}^{-1}\right)$. Hence, $\mu_{1}$ is much larger than $\mu_{0}$ and the boundary layer in the vicinity of $x=1$ is stronger. Consequently, there is a layer of width $O\left(\varepsilon_{2}\right)$ at the left endpoint (the one that arose from the analysis of the case $\varepsilon_{1}=0$ ) and additionally, there is another layer at the right endpoint, of width $O\left(\varepsilon_{1} / \varepsilon_{2}\right)$.

(b) In the regime $\varepsilon_{1} \approx \varepsilon_{2}^{2}$, there are layers at both endpoints of width $O\left(\varepsilon_{1}^{1 / 2}\right)$.

(c) In the regime $\varepsilon_{2}^{2} \ll \varepsilon_{1} \ll 1$, there are layers at both endpoints of width $O\left(\varepsilon_{1}^{1 / 2}\right)$.

It was shown in [9, p. 46] (see also [14, Lemma 2.2], which is the result we quote below) that under the assumptions $b, c, f \in C^{q}(I)$ for some $q \geq 1$ and $q\left\|b^{\prime}\right\|_{\infty, I} \varepsilon_{2} \leq C(1-\ell)$ for some $C, \ell \in(0,1)$, the solution $u$ to (2.1), (2.2) can be decomposed into a smooth part $E_{S}$, a boundary layer part at the left endpoint $E_{L}$, and a boundary layer part at the right endpoint $E_{R}$, viz.

$$
u=E_{S}+E_{L}+E_{R}
$$

with

$$
\left|E_{S}^{(n)}(x)\right| \leq C,\left|E_{L}^{(n)}(x)\right| \leq C \mu_{0}^{n} e^{-\ell \mu_{0} x},\left|E_{R}^{(n)}(x)\right| \leq C \mu_{1}^{n} e^{-\ell \mu_{1}(1-x)},
$$


for all $x \in \bar{I}$ and for $n=0,1,2, \ldots, q$. If one wants to approximate the solution using a fixed order method (e.g., the $h$ version of the Finite Element Method (FEM)), then this regularity result is sufficient for proving convergence; for a higher order method a more refined regularity result is needed for the smooth part. To this end, we assume that $b, c, f$ satisfy, $\forall n=0,1,2, \ldots$,

$$
\left\|f^{(n)}\right\|_{\infty, I} \leq C n ! \gamma_{f}^{n},\left\|c^{(n)}\right\|_{\infty, I} \leq C n ! \gamma_{c}^{n},\left\|b^{(n)}\right\|_{\infty, I} \leq C n ! \gamma_{b}^{n},
$$

for some positive constants $C, \gamma_{f}, \gamma_{c}, \gamma_{b}$, independent of $\varepsilon_{1}, \varepsilon_{2}$. In terms of classical differentiability, we have that the solution to (2.1), (2.2) satisfies (see, e.g., [9, p. 39])

$$
\|u\|_{\infty, I} \leq C
$$

The following lemma provides an estimate for $u^{\prime}$.

LEMMA 2.1. Let $u$ be the solution of (2.1), (2.2) and assume that (2.3), (2.6) hold. Then there exists a positive constant $C$, such that

$$
\left\|u^{\prime}\right\|_{\infty, I} \leq C \max \left\{\varepsilon_{1}^{-1}, \varepsilon_{2}^{-1}\right\} .
$$

Proof. The proof follows [11]. Let

$$
A(x)=\frac{\varepsilon_{2}}{\varepsilon_{1}} \int_{x}^{1} b(t) d t
$$

and note that $A(1)=0$ and $A^{\prime}(x)=-\frac{\varepsilon_{2}}{\varepsilon_{1}} b(x)$. Then multiplying (2.1) by $e^{A(x)}$ and integrating from $x$ to 1 gives

$$
-\varepsilon_{1} u^{\prime}(1)+\varepsilon_{1} e^{A(x)} u^{\prime}(x)+\int_{x}^{1} e^{A(t)} c(t) u(t) d t=\int_{x}^{1} e^{A(t)} f(t) d t .
$$

Multiplying by $e^{-A(x)}$ yields

$$
u^{\prime}(x)=e^{-A(x)} u^{\prime}(1)-\frac{1}{\varepsilon_{1}} \int_{x}^{1} e^{A(t)-A(x)} c(t) u(t) d t+\frac{1}{\varepsilon_{1}} \int_{x}^{1} e^{A(t)-A(x)} f(t) d t .
$$

Integrating from 0 to 1 , we further get

$$
0=u^{\prime}(1) \int_{0}^{1} e^{-A(x)} d x-\frac{1}{\varepsilon_{1}} \int_{0}^{1} \int_{x}^{1} e^{A(t)-A(x)}(c(t) u(t)-f(t)) d t d x .
$$

Since we wish to first estimate $u^{\prime}(1)$, we need upper and lower bounds for $\int_{0}^{1} e^{-A(x)} d x$. From (2.3) we have

$$
\int_{0}^{1} e^{-A(x)} d x \leq \int_{0}^{1} e^{-\frac{\varepsilon_{2}}{\varepsilon_{1}} \beta(x-1)} d x \leq C \frac{\varepsilon_{1}}{\varepsilon_{2} \beta}
$$

Similarly,

$$
\int_{0}^{1} e^{-A(x)} d x \geq \int_{0}^{1} e^{-\frac{\varepsilon_{2}}{\varepsilon_{1}}\|b\|_{\infty, I}(1-x)} d x \geq \frac{\varepsilon_{1}}{\varepsilon_{2}\|b\|_{\infty, I}}\left(1-e^{-\frac{\varepsilon_{2}}{\varepsilon_{1}}\|b\|_{\infty, I}}\right) .
$$

Also, to estimate the remaining term in (2.9), we consider

$$
\frac{1}{\varepsilon_{1}} \int_{0}^{1} \int_{x}^{1} e^{A(t)-A(x)} d t d x=\frac{1}{\varepsilon_{1}} \int_{0}^{1} \int_{x}^{1} e^{A^{\prime}(\zeta)(t-x)} d t d x,
$$


for some $\zeta$ between $t$ and $x$. Hence,

$$
\frac{1}{\varepsilon_{1}} \int_{0}^{1} \int_{x}^{1} e^{A(t)-A(x)} d t d x \leq \frac{1}{\varepsilon_{1}} \int_{0}^{1} \int_{x}^{1} e^{-\frac{\varepsilon_{2}}{\varepsilon_{1}} \beta(t-x)} d t d x \leq C \frac{1}{\varepsilon_{2}}+\frac{\varepsilon_{1}}{\varepsilon_{2}^{2}}
$$

Using (2.9)-(2.11), we get

$$
\begin{aligned}
\left|u^{\prime}(1)\right| & \leq C \frac{1}{\int_{0}^{1} e^{-A(x)} d x}\left(\|c\|_{\infty, I}\|u\|_{\infty, I}+\|f\|_{\infty, I}\right)\left(\frac{1}{\varepsilon_{2}}+\frac{\varepsilon_{1}}{\varepsilon_{2}^{2}}\right) \\
& \leq C \varepsilon_{2} \frac{\|b\|_{\infty, I}}{\varepsilon_{1}}\left(1-e^{-\frac{\varepsilon_{2}}{\varepsilon_{1}}\|b\|_{\infty, I}}\right)^{-1}\left(\frac{1}{\varepsilon_{2}}+\frac{\varepsilon_{1}}{\varepsilon_{2}^{2}}\right) \leq C\left(\varepsilon_{1}^{-1}+\varepsilon_{2}^{-1}\right) .
\end{aligned}
$$

Inserting this bound in (2.8) gives

$$
\begin{aligned}
\left|u^{\prime}(x)\right| & \leq C\left(\varepsilon_{1}^{-1}+\varepsilon_{2}^{-1}\right)+\frac{1}{\varepsilon_{1}}\left(\|c\|_{\infty, I}\|u\|_{\infty, I}+\|f\|_{\infty, I}\right) \int_{x}^{1} e^{A(t)} d t \\
& \leq C\left(\varepsilon_{1}^{-1}+\varepsilon_{2}^{-1}\right)+\frac{1}{\varepsilon_{1}}\left(\|c\|_{\infty, I}\|u\|_{\infty, I}+\|f\|_{\infty, I}\right) \int_{x}^{1} e^{\frac{\varepsilon_{2}}{\varepsilon_{1}}\|b\|_{\infty, I}(1-t)} d t \\
& \leq C\left(\varepsilon_{1}^{-1}+\varepsilon_{2}^{-1}\right)+\frac{1}{\varepsilon_{1}}\left(1-e^{-\frac{\varepsilon_{2}}{\varepsilon_{1}}\|b\|_{\infty, I}}\right) \leq C\left(\varepsilon_{1}^{-1}+\varepsilon_{2}^{-1}\right)
\end{aligned}
$$

as desired.

Using an inductive argument we are able to prove the following.

THEOREM 2.2. Let $u$ be the solution of (2.1), (2.2). Then there exist positive constants $K, C$, independent of $\varepsilon_{1}, \varepsilon_{2}$, and $u$, such that for $n=0,1,2, \ldots$

$$
\left\|u^{(n)}\right\|_{\infty, I} \leq C K^{n} \max \left\{n, \varepsilon_{1}^{-1}, \varepsilon_{2}^{-1}\right\}^{n} .
$$

Proof. The proof is by induction on $n$ and follows from [10]. Equation (2.7) and Lemma 2.1 give the result for $n=0,1$, so we assume it holds for $0 \leq \nu \leq n+1$ and show that it holds for $n+2$. Differentiating $n$ times (2.1) gives

$$
\begin{aligned}
-\varepsilon_{1} u^{(n+2)} & =f^{(n)}-\varepsilon_{2}\left(b u^{\prime}\right)^{(n)}-(c u)^{(n)} \\
& =f^{(n)}-\sum_{\nu=0}^{n}\left(\begin{array}{l}
n \\
\nu
\end{array}\right)\left(\varepsilon_{2} b^{(\nu)} u^{(n+1-\nu)}+c^{(\nu)} u^{(n-\nu)}\right) .
\end{aligned}
$$

By the induction hypothesis we have

$$
\begin{aligned}
\varepsilon_{1}\left\|u^{(n+2)}\right\|_{\infty, I} \leq C & \left\|f^{(n)}\right\|_{\infty, I}+ \\
& +C \sum_{\nu=0}^{n}\left(\begin{array}{l}
n \\
\nu
\end{array}\right)\left(\varepsilon_{2} \gamma_{b}^{\nu} \nu ! K^{n+1-\nu} \max \left\{n+1-\nu, \varepsilon_{1}^{-1}, \varepsilon_{2}^{-1}\right\}^{n+1-\nu}\right. \\
& \left.+\gamma_{c}^{\nu} \nu ! K^{n-\nu} \max \left\{n-\nu, \varepsilon_{1}^{-1}, \varepsilon_{2}^{-1}\right\}^{n-\nu}\right) .
\end{aligned}
$$

Using the estimates below (which follow by standard considerations)

$$
\left(\begin{array}{l}
n \\
\nu
\end{array}\right) \nu ! \max \left\{n+1-\nu, \varepsilon_{1}^{-1}, \varepsilon_{2}^{-1}\right\}^{n+1-\nu} \leq \max \left\{n+1, \varepsilon_{1}^{-1}, \varepsilon_{2}^{-1}\right\}^{n+1}
$$




$$
\begin{gathered}
\left(\begin{array}{l}
n \\
\nu
\end{array}\right) \nu ! \max \left\{n-\nu, \varepsilon_{1}^{-1}, \varepsilon_{2}^{-1}\right\}^{n-\nu} \leq \max \left\{n+1, \varepsilon_{1}^{-1}, \varepsilon_{2}^{-1}\right\}^{n+1} \\
\left\|f^{(n)}\right\|_{\infty, I} \leq C \gamma_{f}^{n} n ! \leq C \max \left\{n+1, \varepsilon_{1}^{-1}, \varepsilon_{2}^{-1}\right\}^{n+1}
\end{gathered}
$$

we obtain

$$
\begin{gathered}
\varepsilon_{1}\left\|u^{(n+2)}\right\|_{\infty, I} \leq C\left\|f^{(n)}\right\|_{\infty, I}+ \\
+C K^{n+2} \max \left\{n+1, \varepsilon_{1}^{-1}, \varepsilon_{2}^{-1}\right\}^{n+1} \sum_{\nu=0}^{n}\left(\frac{1}{K}\left(\frac{\gamma_{b}}{K}\right)^{\nu}+\frac{1}{K^{2}}\left(\frac{\gamma_{c}}{K}\right)^{\nu}\right) \\
\leq C K^{n+2} \max \left\{n+1, \varepsilon_{1}^{-1}, \varepsilon_{2}^{-1}\right\}^{n+1}\left(\frac{1}{K^{2}}+\frac{1}{K} \frac{1}{\left(1-\gamma_{b} / K\right)}+\frac{1}{K^{2}} \frac{1}{\left(1-\gamma_{c} / K\right)}\right) .
\end{gathered}
$$

Choose the constant $K>\max \left\{1, \gamma_{f}, \gamma_{b}, \gamma_{c}\right\}$ such that the expression in brackets above is bounded by 1 , and we have

$$
\varepsilon_{1}\left\|u^{(n+2)}\right\|_{\infty, I} \leq C K^{n+2} \max \left\{n+1, \varepsilon_{1}^{-1}, \varepsilon_{2}^{-1}\right\}^{n+1} .
$$

Dividing by $\varepsilon_{1}$, gives the desired result.

For simplicity, we will focus on the case

$$
\varepsilon_{1}^{2} \ll \varepsilon_{2} .
$$

For the remainder of the article we will make the following assumption:

Assumption 1: Assume there exist positive constants $C, \bar{K}, K_{1}, \ell, K_{2}, \delta>0$, independent of $\varepsilon_{1}, \varepsilon_{2}$, such that the solution $u$ of (2.1), (2.2) can be decomposed into a smooth part $u_{S}$, two boundary layers at each endpoint $u_{B L}^{ \pm}$, and a remainder $u_{R}$, viz.

$$
u=u_{S}+u_{B L}^{-}+u_{B L}^{+}+u_{R},
$$

with the following estimates: for every $n \in \mathbb{N}_{0}$ there holds

$$
\begin{gathered}
\left\|u_{S}^{(n)}\right\|_{\infty, I} \leq C \bar{K}^{n} n !, \\
\left\{\begin{array}{c}
\left|\left(u_{B L}^{-}\right)^{(n)}(x)\right| \leq C K_{1}^{n} \varepsilon_{2}^{-n} e^{-\ell x / \varepsilon_{2}}, \\
\left|\left(u_{B L}^{+}\right)^{(n)}(x)\right| \leq C K_{2}^{n}\left(\frac{\varepsilon_{1}}{\varepsilon_{2}}\right)^{-n} e^{-\ell(1-x) \varepsilon_{2} / \varepsilon_{1}},
\end{array}\right. \\
\left\|u_{R}\right\|_{\infty, \partial I}+\left\|u_{R}\right\|_{0, I}+\varepsilon_{1}^{1 / 2}\left\|u_{R}^{\prime}\right\|_{0, I} \leq C \max \left\{e^{-\delta \varepsilon_{2} / \varepsilon_{1}}, e^{-\delta / \varepsilon_{2}}\right\},
\end{gathered}
$$

for all $x \in \bar{I}$.

We provide in the Appendix, for the convenience of the reader, a proof of Assumption 1 in the case of constant coefficients, in order to illustrate the procedure for obtaining such results. The case of variable coefficients is considerably more tedious (cf. [16]). 
3. Discretization using isogeometric analysis. Isogeometric analysis may be combined with a number of formulations; we use Galerkin's approach, i.e., we multiply (2.1) by a suitable test function, integrate by parts and use the boundary conditions (2.2). The resulting variational formulation reads: find $u \in H_{0}^{1}(I)$ such that

$$
\mathcal{B}(u, v)=\langle f, v\rangle_{I} \quad \forall v \in H_{0}^{1}(I),
$$

where

$$
\mathcal{B}(u, v)=\varepsilon_{1}\left\langle u^{\prime}, v^{\prime}\right\rangle_{I}+\varepsilon_{2}\left\langle b u^{\prime}, v\right\rangle_{I}+\langle c u, v\rangle_{I} .
$$

The bilinear form $\mathcal{B}(\cdot, \cdot)$ given by (3.2) is coercive (due to (2.3)) with respect to the energy norm

$$
\|v\|_{E, I}^{2}:=\varepsilon_{1}|v|_{1, I}^{2}+\rho\|v\|_{0, I}^{2}
$$

i.e.,

$$
\mathcal{B}(v, v) \geq\|v\|_{E, I}^{2} \quad \forall v \in H_{0}^{1}(I) .
$$

Next, we restrict our attention to a finite dimensional subspace $V_{N} \subset H_{0}^{1}(I)$, that will be selected shortly, and obtain the discrete version of (3.1) as: find $u_{N} \in V_{N}$ such that

$$
\mathcal{B}\left(u_{N}, v\right)=\langle f, v\rangle_{I} \quad \forall v \in V_{N}
$$

There holds [9]

$$
\mathcal{B}\left(u_{N}-u, v\right)=0 \quad \forall v \in V_{N} .
$$

In order to define the space $V_{N}$, we first review the concept of IGA. In this article we use B-splines as basis functions and follow [3] closely. To this end let $\Xi=\left\{\xi_{1}, \xi_{2}, \ldots, \xi_{n+p+1}\right\}$ be a knot vector, where $\xi_{i} \in \mathbf{R}$ is the $i$ th knot, $i=1,2, \ldots, N+p+1, p$ is the polynomial order, and $N$ is the number of basis functions. The numbers in $\Xi$ are non-decreasing and may be repeated, in which case we are talking about a non-uniform knot vector. If the first and last knot values appear $p+1$ times, the knot vector is called open (see [3] for more details). With a knot vector $\Xi$ in hand, the B-spline basis functions are defined recursively, starting with piecewise constants $(p=0)$ :

$$
B_{i, 0}(\xi)=\left\{\begin{array}{lc}
1, & \xi_{i} \leq \xi<\xi_{i+1} \\
0, & \text { otherwise }
\end{array}\right.
$$

For $p=1,2, \ldots$, they are defined by the Cox-de Boor recursion formula $[5,6]$ :

$$
B_{i, p}(\xi)=\frac{\xi-\xi_{i}}{\xi_{i+p}-\xi_{i}} B_{i, p-1}(\xi)+\frac{\xi-\xi_{i}}{\xi_{i+p}-\xi_{i}} B_{i, p-1}(\xi) .
$$

We also mention the recursive formula for obtaining the derivative of a B-spline [3]:

$$
\frac{d}{d \xi} B_{i, p}(\xi)=\frac{p}{\xi_{i+p}-\xi_{i}} B_{i, p-1}(\xi)-\frac{p}{\xi_{i+p+1}-\xi_{i+1}} B_{i+1, p-1}(\xi) .
$$

We will be considering open knot vectors, having $\xi_{1}, \ldots, \xi_{m}$ distinct knots, each with multiplicity $r_{i}$. Then

$$
\Xi=[\underbrace{\xi_{1}, \ldots, \xi_{1}}_{r_{1} \text { times }}, \underbrace{\xi_{2}, \ldots, \xi_{2}}_{r_{2} \text { times }}, \ldots, \underbrace{\xi_{m}, \ldots, \xi_{m}}_{r_{m} \text { times }}]
$$


and there holds $\sum_{i=1}^{m} r_{i}=N+p+1$. Since we are using open knots, we have $r_{1}=r_{m}=p+1$. The regularity of the B-spline at each knot $\xi_{i}$ is determined by $r_{i}$, in that the B-spline has $p-r_{i}$ continuous derivatives at $\xi_{i}$. For this reason, we define $k_{i}=p-r_{i}+1$ as a measure of the regularity at the knot $\xi_{i}$ and set $\boldsymbol{k}=\left[k_{1}, \ldots, k_{m}\right]$. Note that $k_{1}=k_{m}=0$ in the case of an open knot vector.

B-splines form a partition of unity and they span the space of continuous piecewise polynomials of degree $p$ on the subdivision $\left\{\xi_{1}, \ldots, \xi_{m}\right\}$.

Each basis function is positive and has support in $\left[\xi_{i}, \xi_{i+p+1}\right]$. In the sections that follow, we will approximate the solution to the BVP under consideration, using the space

$$
S_{\boldsymbol{k}}^{p}=\operatorname{span}\left\{B_{k, p}\right\}_{k=1}^{N}
$$

of dimension

$$
\mathcal{N}=\operatorname{dim}\left(S_{\boldsymbol{k}}^{p}\right)=m p-\sum_{i=1}^{m} k_{i}
$$

We point out that we are using a uniform polynomial degree $p$, while we allow for the regularity at each knot to (possibly) vary. A more general approach would be to allow $p$ to vary as well. We will refer to $\mathcal{N}$ as the number of degrees of freedom, DOF.

Returning to our problem, the space $V_{N}$ in (3.5) is chosen as $V_{N}=S_{\boldsymbol{k}}^{p}$, given by (3.6). Thus, we may write the approximate solution as

$$
u_{N}=\sum_{k=0}^{N} \alpha_{k} B_{k, p}
$$

with $\alpha=\left[\alpha_{1}, \ldots, \alpha_{N}\right]^{T}$ unknown coefficients, and subsitute in (3.5) to obtain the linear system of equations

$$
\underbrace{\left(\varepsilon_{1} A_{1}+\varepsilon_{2} A_{2}+A_{0}\right)}_{\mathbf{M} \in \mathbb{R}^{N \times N}} \alpha=\mathbf{f},
$$

where

$$
\begin{gathered}
{\left[A_{1}\right]_{i, j}=\int_{I} B_{i, p}^{\prime}(\xi) B_{j, p}^{\prime}(\xi) d \xi,\left[A_{2}\right]_{i, j}=\int_{I} B_{i, p}^{\prime}(\xi) B_{j, p}(\xi) d \xi} \\
{\left[A_{0}\right]_{i, j}=\int_{I} B_{i, p}(\xi) B_{j, p}(\xi) d \xi,[\mathbf{f}]_{i}=\int_{I} B_{i, p}(\xi) f(\xi) d \xi}
\end{gathered}
$$

for $i, j=1, \ldots, N$. The linear system (3.7) has a unique solution since the matrix $\mathbf{M}$ is invertible (due to (3.4) and the linear independence of the basis functions $B_{j, p}$ ).

If $\varepsilon_{1}$ and $\varepsilon_{2}$ are large, then no boundary layers are present and approximating $u$ may be done using a fixed mesh (of say one element) and increasing $p$. On the other hand, if $\varepsilon_{1}$ and $\varepsilon_{2}$ are small, then classical techniques fail and the mesh must be chosen carefully. The challenge lies in approximating the typical boundary layer function $e^{-x / \varepsilon}$. In the context of FDs and FEs, the mesh points must depend on $\varepsilon$, as is well documented in the literature under the name layer-adapted meshes [9]. A similar observation holds for IGA, in the sense that the knot vector must depend on $\varepsilon$. This was illustrated in [8] through numerical experiments and in the next section we establish it mathematically. 
4. Error estimates. We begin by citing the main tool for the analysis (see Corollary 2 in [4]).

PROPOSITION 4.1. Given the subdivision $\left\{0=\xi_{1}, \ldots, \xi_{m}=1\right\}$ of the reference domain $I=(0,1)$, let $I_{i}=\left(\xi_{i}, \xi_{i+1}\right), h_{i}=\xi_{i+1}-\xi_{i}, i=1, \ldots, m-1, k, p$ non-negative integers with $p \geq 2 k-1$ and $u^{(k)} \in H^{s}(I)$ for some $0 \leq s \leq \kappa=p-k+1$. Then there exists a quasi-interpolation operator $\pi_{p, k}$ such that, for $i=1, \ldots, m-1, j=1, \ldots, k-1$,

$$
\left\|u^{(j)}-\left(\pi_{p, k} u\right)^{(j)}\right\|_{L^{2}\left(I_{i}\right)}^{2} \leq\left(\frac{h_{i}}{2}\right)^{2(s+k-j)} \frac{(\kappa-s) !(\kappa-(k-j)) !}{(\kappa+s) !(\kappa+(k-j)) !}\left|u^{(k)}\right|_{H^{s}\left(I_{i}\right)}^{2} .
$$

In [2], the above was specialized for maximal smoothness by selecting $p=2 q-1$, for some $q \geq 1$, and then $k=\kappa=q$, so the estimate of Proposition 4.1 becomes

$$
\left\|u^{(j)}-\left(\pi_{2 q-1, q} u\right)^{(j)}\right\|_{L^{2}\left(I_{i}\right)}^{2} \leq\left(\frac{h_{i}}{2}\right)^{2(s+q-j)} \frac{(q-s) ! j !}{(q+s) !(2 q-j) !}\left\|u^{(q+s)}\right\|_{L^{2}\left(I_{i}\right)}^{2},
$$

for $0 \leq s \leq q, j=1, \ldots, q-1$.

In view of the above, from now on we will be using the symbol $q$ to denote the polynomial degree.

The knot vector described below, is 'inspired' by the so-called spectral boundary layer $m e s h$ used in the $h p$-FEM for such problems (see [12] and the references therein).

DEFINITION 4.2. Let $\mu_{0}, \mu_{1}$ be given by (2.4) and let $\lambda \geq 1$ be a user specified parameter. Then, if $\lambda q \mu_{1}^{-1} \geq 1 / 2$,

$$
\Xi=[\underbrace{0, \ldots, 0}_{q+1 \text { times }}, \underbrace{1, \ldots, 1}_{q+1 \text { times }}]
$$

and, if $\lambda q \mu_{0}^{-1}<1 / 2$,

$$
\Xi=[\underbrace{0, \ldots, 0}_{q+1 \text { times }}, \lambda q \mu_{0}^{-1}, 1-\lambda q \mu_{1}^{-1}, \underbrace{1, \ldots, 1}_{q+1 \text { times }}],
$$

where $q$ is the polynomial degree.

The following auxiliary result will be used in the sequel.

LEMMA 4.3. There exists $\tau \in[1 / 2,2 / 3]$ such that for every $q \geq 2,3,4, \ldots$, there holds $s:=\tau q \in \mathbb{N}$ and

$$
\frac{(q-s) !}{(q+s) !} \leq C e^{-\sigma q} q^{-2 \tau q} e^{2 \tau q}
$$

where

$$
\sigma:=\min _{\tau \in[1 / 2,2 / 3]}\left|\ln \frac{(1-\tau)^{1-\tau}}{(1+\tau)^{1+\tau}}\right| .
$$

Proof. We first show $\tau q \in \mathbb{N}$, for some $\tau \in[1 / 2,2 / 2]$. If $q$ is even, then we simply take $\tau=1 / 2$. If $q$ is odd, i.e., $q=2 n+1, n \in \mathbb{N}$, then we choose $\tau=(n+1) /(2 n+1)$.

Now, with $\tau q \in \mathbb{N}$, we have $(q \pm \tau q) !=\Gamma(q \pm \tau q+1)$ and, as $q \rightarrow \infty$ [1],

$$
\begin{aligned}
\frac{(q-\tau q) !}{(q+\tau q) !} & =\frac{\Gamma(q-\tau q+1)}{\Gamma(q+\tau q+1)} \leq C \frac{(q(1-\tau)+1)^{q-\tau q+1 / 2} e^{-(q-\tau q+1)}}{(q(1+\tau)+1)^{q+\tau q+1 / 2} e^{-(q+\tau q+1)}} \\
& \leq C\left(\frac{(1-\tau)^{1-\tau}}{(1+\tau)^{1+\tau}}\right)^{q} q^{-2 \tau q} e^{2 \tau q} \leq C e^{-\sigma q} q^{-2 \tau q} e^{2 \tau q}
\end{aligned}
$$


THEOREM 4.4. Let $u$ be the solution to (2.1), (2.2) and let $\pi_{2 q-1, q} u \in S_{q}^{2 q-1}$ be its quasi-interpolant, constructed using the knot vector of Definition 4.2 and satisfying (4.1). Then there exist positive constants $\sigma, C$, independent of $\varepsilon_{1}, \varepsilon_{2}$, such that as $q \rightarrow \infty$ there holds

$$
\left\|u-\pi_{2 q-1, q} u\right\|_{E, I} \leq C e^{-\sigma q} .
$$

Proof. The proof is separated into two cases. In Case 1, we make use of the classical differentiability result (2.12) and construct a quasi-interpolant of $u$ on the entire interval $I$ with the desired properties. In Case 2, we use the decomposition (2.13) and construct quasiinterpolants for each piece separately, as follows: for the smooth part, the quasi-interpolant is constructed on $I$, while for the boundary layers, it has support only in the layer regions. No quasi-interpolant is constructed for the remainder, since it is already exponentially small. We next give the details.

Case 1: $\lambda q \mu_{1}^{-1} \geq 1 / 2$ or equivalently $2 \lambda q \geq \varepsilon_{1}^{-1}$. In this case, the knot vector is given by (4.2). We make use of (2.12) and (4.1) to obtain a quasi-interpolant $\pi_{2 q-1, q} u$ on $I$, such that

$$
\begin{aligned}
\left\|u-\pi_{2 q-1, q} u\right\|_{E, I}^{2} & \leq C \frac{(q-s) !}{(q+s) !}\left(\frac{\varepsilon_{1}}{(2 q-1) !}+\frac{\rho}{(2 q) !}\right)\left\|u^{(q+s)}\right\|_{L^{2}(I)}^{2} \\
& \leq C \frac{(q-s) !}{(q+s) !}\left(\frac{2 q \varepsilon_{1}+\rho}{(2 q) !}\right) K^{2(q+s)} \max \left\{\varepsilon_{1}^{-1}, q+s\right\}^{2(q+s)} .
\end{aligned}
$$

We choose $s=\tau q$, with $\tau \in[1 / 2,2 / 3]$ as asserted by Lemma 4.3, and we note that, from (2.5) and the fact that in this case there holds $2 \lambda q \geq \varepsilon_{1}^{-1}$, we have

$$
\begin{aligned}
\max \left\{\varepsilon_{1}^{-1}, q+s\right\}^{2(q+s)} & =\max \left\{\varepsilon_{1}^{-1},(\tau+1) q\right\}^{2 q(\tau+1)} \\
& \leq \max \{2 \lambda q,(\tau+1) q\}^{2 q(\tau+1)} \leq(2 \lambda q)^{2 q(\tau+1)},
\end{aligned}
$$

since $2 \lambda \geq \tau+1$. Then, with the aid of Lemma 4.3, we get

$$
\begin{aligned}
\left\|u-\pi_{2 q-1, q} u\right\|_{E, I}^{2} & \leq C \frac{(q-\tau q) !}{(q+\tau q) !}\left(\frac{2 q \varepsilon_{1}+\rho}{(2 q) !}\right) K^{2(\tau+1) q}(2 \lambda q)^{2 q(\tau+1)} \\
& \leq C e^{-\sigma_{1} q} q^{-2 \tau q} e^{2 \tau q+1}\left(\frac{2 q \varepsilon_{1}+\rho}{(2 q) !}\right) K^{2(\tau+1) q}(2 \lambda q)^{2 q(\tau+1)} \\
& \leq C \frac{q^{2 q+1}}{(2 q) !} K^{2 q} e^{-\sigma_{1} q}(2 e K \lambda)^{2 \tau q}
\end{aligned}
$$

with $\sigma_{1}$ given by (4.4). By Stirling's formula $\frac{q(K q)^{2 q}}{(2 q) !} \leq \sqrt{q}(e K / 2)^{2 q}$, hence

$$
\begin{aligned}
\left\|u-\pi_{2 q-1, q} u\right\|_{E, I}^{2} & \leq C \sqrt{q}(e K / 2)^{2 q} e^{-\sigma_{1} q}(2 e K \lambda)^{2 \tau q} \\
& \leq C \sqrt{q} e^{-\sigma_{1} q}\left((2 e K \lambda)^{2 \tau}(e K / 2)^{2}\right)^{q} .
\end{aligned}
$$

Selecting $\lambda$ such that $(2 e K \lambda)^{2 \tau}(e K / 2)^{2}<1$, gives

$$
\left\|u-\pi_{2 q-1, q} u\right\|_{E, I} \leq C q^{1 / 4} e^{-\sigma_{1} q},
$$

where $C>0$ is independent of $q \in \mathbb{N}$ and $\varepsilon_{1}$. Reducing the value of $\sigma_{1}$ to $\tilde{\sigma}_{1} \in\left(0, \sigma_{1}\right)$, there exists a positive constant $C$, independent of $q$ and $\varepsilon_{1}$, such that

$$
\left\|u-\pi_{2 q-1, q} u\right\|_{E, I} \leq C e^{-\tilde{\sigma_{1}} q} .
$$


Case 2: $\lambda q \mu_{0}^{-1}<1 / 2$ or equivalently, $2 q \varepsilon_{1}<1 / \lambda$. In this case, the solution is decomposed as in (2.13) with the bounds (2.14)-(2.16) being valid. We will approximate each term in (2.13) separately, using Proposition 4.1 to construct appropriate quasi-interpolants over the three intervals

$$
I_{1}=\left(0, q \mu_{0}^{-1}\right), I_{2}=\left(q \mu_{0}^{-1}, 1-q \mu_{1}^{-1}\right), I_{3}=\left(q \mu_{1}^{-1}, 1\right) .
$$

Throughout the proof, we choose

$$
\lambda \leq \frac{2}{e} \max \left\{\frac{1}{K}, \frac{1}{K_{1}}, \frac{1}{K_{2}}\right\},
$$

with $K, K_{1}, K_{2}$ the constants in (2.14)-(2.16). So we have, with the obvious notation,

$$
u-\pi_{2 q-1, q} u=u_{S}+u_{B L}^{-}+u_{B}^{+}+u_{R}-\pi_{2 q-1, q} u_{S}-\pi_{2 q-1, q} u_{B L}^{-}-\pi_{2 q-1, q} u_{B L}^{+},
$$

hence

$$
\left|u-\pi_{2 q-1, q} u\right| \leq\left|u_{S}-\pi_{2 q-1, q} u_{S}\right|+\left|u_{B L}^{-}-\pi_{2 q-1, q} u_{B L}^{-}\right|+\left|u_{B}^{+}-\pi_{2 q-1, q} u_{B L}^{+}\right|+\left|u_{R}\right| .
$$

For the smooth part, Proposition 4.1 ensures that $\pi_{2 q-1, q} u_{S}$, is such that

$$
\begin{aligned}
\left\|u_{S}-\pi_{2 q-1, q} u_{S}\right\|_{E, I}^{2} & \leq C \frac{(q-s) !}{(q+s) !}\left(\frac{\varepsilon_{1}}{(2 q-1) !}+\frac{\rho}{(2 q) !}\right)\left\|u_{S}^{(q+s)}\right\|_{L^{2}(I)}^{2} \\
& \leq C \frac{(q-s) !}{(q+s) !} \frac{1}{\lambda(2 q) !} K^{2(q+s)}((2(q+s)) !)^{2} .
\end{aligned}
$$

Choosing $s=\widetilde{\tau} q$ with $\widetilde{\tau} \in[1 / 2,2 / 3]$ as in Lemma 4.3, and using Lemma 1 of [2], we get

$$
\begin{aligned}
\left\|u_{S}-\pi_{2 q-1, q} u_{S}\right\|_{E, I}^{2} & \leq C\left(\frac{1}{4} \frac{(1+\widetilde{\tau})^{1+\widetilde{\tau}}}{(1-\widetilde{\tau})^{\tau}-1}\right)^{q} \frac{1}{\lambda(2 q) !} K^{2(q+\widetilde{\tau} q)} \\
& \leq C\left(\frac{1}{4} \frac{(1+\widetilde{\tau})^{1+\widetilde{\tau}}}{(1-\widetilde{\tau})^{\widetilde{\tau}-1}}\right)^{q} \frac{1}{\lambda(2 q)^{2 q+1 / 2} e^{-2 q}} K^{2(q+\widetilde{\tau} q)} \\
& \leq C \frac{1}{\lambda(2 q)^{1 / 2}}\left(\frac{1}{4} \frac{(1+\widetilde{\tau})^{1+\widetilde{\tau}}}{(1-\widetilde{\tau})^{\tau}-1}\right)^{q}\left(\frac{e K^{\widetilde{\tau}+1}}{2 q}\right)^{2 q} \\
& \leq C\left(\frac{K}{\lambda q}\right)^{2 q}
\end{aligned}
$$

by (4.7). Therefore, there exist constants $C, \sigma_{2}>0$ such that for every $\varepsilon_{1}, \varepsilon_{2} \in(0,1]$, and for every $q \in \mathbb{N}$, there holds

$$
\left\|u_{S}-\pi_{2 q-1, q} u_{S}\right\|_{E, I} \leq C e^{-\sigma_{2} q} .
$$

For the left boundary layer $u_{B L}^{-}$we obtain from Proposition 4.1, a quasi-interpolant $\pi_{2 q-1, q} u_{B L}^{-}$on the interval $I_{1}=\left(0, \lambda q \mu_{0}^{-1}\right)$, such that

$$
\begin{aligned}
\left|u_{B L}^{-}-\pi_{2 q-1, q} u_{B L}^{-}\right|_{1, I_{1}}^{2} & \leq C\left(\frac{\lambda q \mu_{0}^{-1}}{2}\right)^{2(s+q-1)} \frac{(q-s) !}{(q+s) !} \frac{2 q}{(2 q-1) !}\left\|\left(u_{B L}^{-}\right)^{(q+s)}\right\|_{L^{2}\left(I_{1}\right)}^{2} \\
& \leq C\left(\frac{\lambda q \mu_{0}^{-1}}{2}\right)^{2(s+q-1)} \frac{(q-s) !}{(q+s) !} \frac{2 q}{(2 q-1) !} K_{1}^{2(q+s)} \varepsilon_{2}^{-2(q+s)+1}
\end{aligned}
$$


Using (2.5), we obtain

$$
\begin{aligned}
\left|u_{B L}^{-}-\pi_{2 q-1, q} u_{B L}^{-}\right|_{1, I_{1}}^{2} & \leq C \mu_{0}\left(\mu_{0} \varepsilon_{2}\right)^{-2(q+s)+1}\left(\frac{\lambda q}{2}\right)^{2 s} \frac{(q-s) !}{(q+s) !} \frac{(\lambda q)^{2 q-2}}{(2 q-1) !} K_{1}^{2(q+s)} \\
& \leq C \varepsilon_{1}^{-1 / 2}(q / e)^{2 s} \frac{(q-s) !}{(q+s) !} \frac{(\lambda q)^{2 q-2}}{(2 q-1) !} K_{1}^{2 q}
\end{aligned}
$$

where (4.7) was used. Choosing $s=\bar{\tau} q$, with $\bar{\tau} \in[1 / 2,2 / 3]$ as in Lemma 4.3, we get (for $\sigma_{3}$ given by (4.4) with $\tau$ replaced by $\bar{\tau}$ ),

$$
\begin{aligned}
\left|u_{B L}^{-}-\pi_{2 q-1, q} u_{B L}^{-}\right|_{1, I_{1}}^{2} & \leq C \varepsilon_{1}^{-1 / 2}\left(e^{-1} q\right)^{2 \bar{\tau} q} e^{-\sigma_{3} q} q^{-2 \bar{\tau} q} e^{2 \bar{\tau} q+1} \frac{(\lambda q)^{2 q-2}}{(2 q-1) !} K_{1}^{2 q} \\
& \leq C \varepsilon_{1}^{-1 / 2} e^{-\sigma_{3} q} \frac{(\lambda q)^{2 q-2}}{(2 q-1) !} K_{1}^{2 q} \\
& \leq C \varepsilon_{1}^{-1 / 2}(\lambda q)^{-2} e^{-\sigma_{3} q} \frac{\left(\lambda q K_{1}\right)^{2 q}}{(2 q-1) !}
\end{aligned}
$$

Since, by (4.7) and Stirling's formula, $\frac{\left(\lambda q K_{1}\right)^{2 q}}{(2 q-1) !} \leq \frac{\left(2 q e^{-1}\right)^{2 q}}{(2 q-1) !} \leq \frac{e^{-2 q}(2 q)^{2 q} 2 q}{(2 q)^{2 q+1 / 2} e^{-2 q}} \leq(2 q)^{1 / 2}$, we have

$$
\left|u_{B L}^{-}-\pi_{2 q-1, q} u_{B L}^{-}\right|_{1, I_{1}} \leq C \varepsilon_{1}^{-1 / 4} e^{-\sigma_{3} q},
$$

with $C>0$ independent of $\varepsilon_{1}$ and $q$. On $I \backslash I_{1}, u_{B L}^{-}$is already exponentially small, i.e., by (2.15), we have

$$
\begin{aligned}
\left|u_{B L}^{-}\right|_{1, I \backslash I_{1}}^{2} & =\int_{q \mu_{0}^{-1}}^{1}\left[\left(u_{B L}^{-}\right)^{\prime}(x)\right]^{2} d x \leq C \varepsilon_{2}^{-2} \int_{q \mu_{0}^{-1}}^{1} e^{-2 \ell q \mu_{0}^{-1} / \varepsilon_{2}} d x \\
& \leq C \varepsilon_{2}^{-1} e^{-\ell \lambda q}
\end{aligned}
$$

hence it will not be approximated. Thus, there exist constants $C, \bar{\sigma}_{3}>0$ such that for every $\varepsilon_{1}, \varepsilon_{2} \in(0,1]$, and for every $q \in \mathbb{N}$, there holds

$$
\begin{aligned}
\left|u_{B L}^{-}-\pi_{2 q-1, q} u_{B L}^{-}\right|_{1, I} & \leq\left|u_{B L}^{-}-\pi_{2 q-1, q} u_{B L}^{-}\right|_{1, I_{1}}+\left|u_{B L}^{-}\right|_{1, I \backslash I_{1}} \\
& \leq C\left(\varepsilon_{1}^{-1 / 4} e^{-\sigma_{3} q}+\varepsilon_{2}^{-1 / 2} e^{-\ell \lambda q}\right) \\
& \leq C \varepsilon_{1}^{-1 / 2} e^{-\bar{\sigma}_{3} q},
\end{aligned}
$$

where again (2.5) was used. For the $L^{2}$ error, we have

$$
\begin{aligned}
\left\|u_{B L}^{-}-\pi_{2 q-1, q} u_{B L}^{-}\right\|_{0, I_{1}}^{2} & \leq\left(\frac{\lambda q \mu_{0}^{-1}}{2}\right)^{2(s+q)} \frac{(q-s) !}{(q+s) !} \frac{1}{(2 q) !}\left\|\left(u_{B L}^{-}\right)^{(q+s)}\right\|_{L^{2}\left(I_{1}\right)}^{2} \\
& \leq C\left(\frac{\lambda q \mu_{0}^{-1}}{2}\right)^{2(s+q)} \frac{(q-s) !}{(q+s) !} \frac{1}{(2 q) !} K_{1}^{2(q+s)} \varepsilon_{2}^{-2(q+s)+1} \\
& \leq C \varepsilon_{2}\left(\mu_{0} \varepsilon_{2}\right)^{-2(q+s)}\left(\frac{\lambda q}{2}\right)^{2(s+q)} \frac{(q-s) !}{(q+s) !} \frac{1}{(2 q) !} K_{1}^{2(q+s)} .
\end{aligned}
$$

Choosing $s$ as a non-integer multiple of $q$, and repeating the same steps as for the $H^{1}$ seminorm, we see that there exist constants $C, \sigma_{4}>0$ such that for every $\varepsilon_{1}, \varepsilon_{2} \in(0,1]$, and for every $q \in \mathbb{N}$, there holds

$$
\left\|u_{B L}^{-}-\pi_{2 q-1, q} u_{B L}^{-}\right\|_{0, I_{1}} \leq C \varepsilon_{2}^{1 / 2} e^{-\sigma_{4} q} .
$$


On $I \backslash I_{1}, u_{B L}^{-}$is already exponentially small, hence there exist constants $C, \sigma_{5}>0$ such that for every $\varepsilon_{1}, \varepsilon_{2} \in(0,1]$, and for every $q \in \mathbb{N}$, there holds

$$
\begin{aligned}
\left\|u_{B L}^{-}-\pi_{2 q-1, q} u_{B L}^{-}\right\|_{0, I} & \leq\left\|u_{B L}^{-}-\pi_{2 q-1, q} u_{B L}^{-}\right\|_{0, I_{1}}+\left\|u_{B L}^{-}\right\|_{0, I \backslash I_{1}} \\
& \leq C\left(\varepsilon_{2}^{1 / 2} e^{-\sigma_{4} q}+e^{-\ell \mu_{0} \lambda q \mu_{0}^{-1}}\right) \\
& \leq C e^{-\sigma_{5} q} .
\end{aligned}
$$

We follow the same steps for the right boundary layer $u_{B L}^{+}$: Proposition 4.1 gives $\pi_{2 q-1, q} u_{B L}^{+}$on $I_{3}$, such that

$$
\begin{aligned}
\mid u_{B L}^{+} & -\left.\pi_{2 q-1, q} u_{B L}^{+}\right|_{1, I_{3}} ^{2} \\
& \leq\left(\frac{\lambda q \mu_{1}^{-1}}{2}\right)^{2(s+q-1)} \frac{(q-s) !}{(q+s) !} \frac{2 q}{(2 q-1) !}\left\|\left(u_{B L}^{+}\right)^{(q+s)}\right\|_{L^{2}\left(I_{3}\right)}^{2} \\
& \leq C\left(\frac{\lambda q \mu_{1}^{-1}}{2}\right)^{2(s+q-1)} \frac{(q-s) !}{(q+s) !} \frac{2 q}{(2 q-1) !} K_{2}^{2(q+s)}\left(\frac{\varepsilon_{1}}{\varepsilon_{2}}\right)^{-2(q+s)+1} \\
& \leq C \mu_{1}\left(\frac{\varepsilon_{2}}{\mu_{1} \varepsilon_{1}}\right)^{2(q+s)+1}\left(\frac{\lambda q}{2}\right)^{2(s+q-1)} \frac{(q-s) !}{(q+s) !} \frac{2 q}{(2 q-1) !} K_{2}^{2(q+s)} .
\end{aligned}
$$

Using (2.5), we obtain

$$
\left|u_{B L}^{+}-\pi_{2 q-1, q} u_{B L}^{+}\right|_{1, I_{3}}^{2} \leq C \varepsilon_{1}^{-1}\left(\frac{q}{e}\right)^{2 s} \frac{(q-s) !}{(q+s) !} \frac{(\lambda q)^{2 q-2}}{(2 q-1) !} K_{2}^{2 q} .
$$

Choosing $s=\widetilde{\tau} q$, with $\widetilde{\tau} \in[1 / 2,2 / 3]$ as in Lemma 4.3, and following the same steps as in the approximation of the left boundary layer (using (4.7)), we arrive at

$$
\left|u_{B L}^{+}-\pi_{2 q-1, q} u_{B L}^{+}\right|_{1, I_{3}} \leq C \varepsilon_{1}^{-1 / 2} e^{-\sigma_{6} q}
$$

with $\sigma_{6}$ given by (4.4) with $\tau$ replaced by $\widetilde{\tau}$. On $I \backslash I_{3}, u_{B L}^{+}$is already exponentially small, i.e., by (2.15) we have

$$
\begin{aligned}
\left|u_{B L}^{+}\right|_{1, I \backslash I_{3}}^{2} & =\int_{0}^{q \mu_{1}^{-1}}\left[\left(u_{B L}^{+}\right)^{\prime}(x)\right]^{2} d x \leq C\left(\frac{\varepsilon_{1}}{\varepsilon_{2}}\right)^{-2} \int_{0}^{q \mu_{1}^{-1}} e^{-\ell(1-x) \varepsilon_{2} / \varepsilon_{1}} d x \\
& \leq C\left(\frac{\varepsilon_{1}}{\varepsilon_{2}}\right)^{-1} e^{-\ell \lambda q},
\end{aligned}
$$

hence it will not be approximated. Thus

$$
\begin{aligned}
\left|u_{B L}^{+}-\pi_{2 q-1, q} u_{B L}^{+}\right|_{1, I} & \leq\left|u_{B L}^{+}-\pi_{2 q-1, q} u_{B L}^{+}\right|_{1, I_{3}}+\left|u_{B L}^{+}\right|_{1, I \backslash I_{3}} \\
& \leq C\left(\varepsilon_{1}^{-1 / 2} e^{-\sigma_{6} q}+\left(\frac{\varepsilon_{1}}{\varepsilon_{2}}\right)^{-1 / 2} e^{-\ell \lambda q}\right) \\
& \leq C \varepsilon_{1}^{-1} e^{-\widetilde{\sigma}_{6} q}
\end{aligned}
$$


for some positive constant $\widetilde{\sigma}_{6}$, where (2.5) was used once more. For the $L^{2}$ error, we have

$$
\begin{aligned}
\left\|u_{B L}^{+}-\pi_{2 q-1, q} u_{B L}^{+}\right\|_{0, I_{3}}^{2} & \leq\left(\frac{\lambda q \mu_{1}^{-1}}{2}\right)^{2(s+q)} \frac{(q-s) !}{(q+s) !} \frac{1}{(2 q) !}\left\|\left(u_{B L}^{+}\right)^{(q+s)}\right\|_{L^{2}\left(I_{3}\right)}^{2} \\
& \leq C\left(\frac{\lambda q \mu_{1}^{-1}}{2}\right)^{2(s+q)} \frac{(q-s) !}{(q+s) !} \frac{1}{(2 q) !} K_{1}^{2(q+s)}\left(\frac{\varepsilon_{1}}{\varepsilon_{2}}\right)^{-2(q+s)+1} \\
& \leq C \frac{\varepsilon_{1}}{\varepsilon_{2}}\left(\frac{\varepsilon_{2}}{\mu_{1} \varepsilon_{1}}\right)^{2(q+s)}\left(\frac{\lambda q}{2}\right)^{2(s+q)} \frac{(q-s) !}{(q+s) !} \frac{1}{(2 q) !} K_{1}^{2(q+s)} \\
& \leq C \frac{\varepsilon_{1}}{\varepsilon_{2}}\left(\frac{\lambda q}{2}\right)^{2(s+q)} \frac{(q-s) !}{(q+s) !} \frac{1}{(2 q) !} K_{1}^{2(q+s)},
\end{aligned}
$$

where we used (2.5) and (4.7). The remaining steps are the same as above, so they are omitted. We arrive at

$$
\left\|u_{B L}^{+}-\pi_{2 q-1, q} u_{B L}^{+}\right\|_{0, I_{3}} \leq C\left(\frac{\varepsilon_{1}}{\varepsilon_{2}}\right)^{1 / 2} e^{-\sigma_{7} q}
$$

for some positive constant $\sigma_{7}$. Finally,

$$
\begin{aligned}
\left\|u_{B L}^{+}-\pi_{2 q-1, q} u_{B L}^{+}\right\|_{0, I} & \leq\left\|u_{B L}^{+}-\pi_{2 q-1, q} u_{B L}^{+}\right\|_{0, I_{3}}+\left\|u_{B L}^{+}\right\|_{0, I \backslash I_{3}} \\
& \leq C\left(\left(\frac{\varepsilon_{1}}{\varepsilon_{2}}\right)^{1 / 2} e^{-\sigma_{7} q}+e^{-\ell \lambda q}\right) \\
& \leq C e^{-\widetilde{\sigma}_{7} q},
\end{aligned}
$$

for some positive constant $\widetilde{\sigma}_{7}$.

It remains to consider the remainder, which by (2.16) is exponentially small:

$$
\left\|u_{R}\right\|_{E, I} \leq C \max \left\{e^{-\delta \varepsilon_{2} / \varepsilon_{1}}, e^{-\delta / \varepsilon_{2}}\right\} \leq C e^{-\delta / \varepsilon_{2}} \leq C e^{-\zeta \lambda q},
$$

since $\lambda p \mu_{0}^{-1}<1 / 2$ (hence $\lambda p \varepsilon_{2}<1 / 2$ ), where $\zeta>0$ is a constant independent of $\varepsilon_{1}, \varepsilon_{2}$.

The proof is completed by using the definition of the energy norm (3.3), along with (2.16), (4.5), (4.8)-(4.14), to get

$$
\begin{aligned}
\left\|u-\pi_{2 q-1, q} u\right\|_{E, I} \leq & \varepsilon_{1}\left(\left|u_{S}-\pi_{2 q-1, q} u_{S}\right|_{1, I}+\left|u_{B L}^{ \pm}-\pi_{2 q-1, q} u_{B L}^{ \pm}\right|_{1, I}\right)+ \\
& \left\|u_{S}-\pi_{2 q-1, q} u_{S}\right\|_{0, I}+\left\|u_{B L}^{ \pm}-\pi_{2 q-1, q} u_{B L}^{ \pm}\right\|_{0, I}+\left\|u_{R}\right\|_{E, I} \\
\leq & C e^{-\sigma q}
\end{aligned}
$$

for some positive consant $\sigma$.

We next estimate the difference between the IGA solution $u_{N}$ and the quasi-interpolant $\pi_{2 q-1, q} u$.

LEMmA 4.5. Let $u_{N} \in S_{q}^{2 q-1}$ be the approximation to $u$, the solution of (2.1), (2.2), based on the knot vector of Defintion 4.2, and let $\pi_{2 q-1, q}$ be the approximation operator of Proposition 4.1. Then there exist constants $C, \sigma>0$, independent of $\varepsilon_{1}, \varepsilon_{2}$, such that $\forall q \in \mathbb{N}$,

$$
\left\|\pi_{2 q-1, q} u-u_{N}\right\|_{E, I} \leq C e^{-\sigma q} .
$$

Proof. Set $\xi:=\pi_{2 q-1, q} u-u_{N}$. Then, by coercivity of the bilinear form $\mathcal{B}_{\varepsilon}$ (eq. (3.4)), there holds

$$
\|\xi\|_{E, I}^{2} \leq \mathcal{B}_{\varepsilon}(\xi, \xi)=-\mathcal{B}_{\varepsilon}\left(u-\pi_{2 q-1, q} u, \xi\right)
$$


where we also used Galerkin orthogonality. Hence,

$\|\xi\|_{E, I}^{2} \leq-\varepsilon_{1}\left\langle\left(u-\pi_{2 q-1, q} u\right)^{\prime}, \xi^{\prime}\right\rangle_{I}+\varepsilon_{2}\left\langle b\left(u-\pi_{2 q-1, q} u\right)^{\prime}, \xi\right\rangle_{I}+\left\langle c\left(u-\pi_{2 q-1, q} u\right), \xi\right\rangle_{I}$.

The first and last term may be estimated using Cauchy-Schwarz:

$$
\begin{aligned}
\mid-\varepsilon_{1} & \left\langle\left(u-\pi_{2 q-1, q} u\right)^{\prime}, \xi^{\prime}\right\rangle_{I}|+|\left\langle c\left(u-\pi_{2 q-1, q} u\right), \xi\right\rangle_{I} \mid \\
& \leq \varepsilon_{1}\left\|\left(u-\pi_{2 q-1, q} u\right)^{\prime}\right\|_{0, I}\left\|\xi^{\prime}\right\|_{0, I}+\|c\|_{\infty, I}\left\|u-\pi_{2 q-1, q} u\right\|_{0, I}\|\xi\|_{0, I} \\
& \leq C \max \left\{1,\|c\|_{\infty, I}\right\}\left\|u-\pi_{2 q-1, q} u\right\|_{E, I}\|\xi\|_{E, I} .
\end{aligned}
$$

For the second term, we will consider the two ranges of $q$ separately: in the asymptotic range of $q$, i.e., $\lambda q \mu_{1}^{-1} \geq 1 / 2$ or equivalently $\lambda q \varepsilon_{1} \geq 1 / 2$, we have

$$
\begin{aligned}
\left|\varepsilon_{2}\left\langle b\left(u-\pi_{2 q-1, q} u\right)^{\prime}, \xi\right\rangle_{I}\right| & \leq C \varepsilon_{2}\|b\|_{\infty, I}\left\|\left(u-\pi_{2 q-1, q} u\right)^{\prime}\right\|_{0, I}\|\xi\|_{0, I} \\
& \leq C \varepsilon_{2} \varepsilon_{1}^{-1 / 2}\left\|u-\pi_{2 q-1, q} u\right\|_{E, I}\|\xi\|_{E, I} \\
& \leq C \varepsilon_{2}(\lambda q)^{1 / 2}\left\|u-\pi_{2 q-1, q} u\right\|_{E, I}\|\xi\|_{E, I} \\
& \leq C e^{-\sigma q}\|\xi\|_{E, I} .
\end{aligned}
$$

In the pre-asymptotic range of $q$, i.e., $\lambda q \mu_{0}^{-1}<1 / 2$, we first use integration by parts to obtain

$$
\left|\varepsilon_{2}\left\langle b\left(u-\pi_{2 q-1, q} u\right)^{\prime}, \xi\right\rangle_{I}\right|=\left|\varepsilon_{2}\left\langle b\left(u-\pi_{2 q-1, q} u\right), \xi^{\prime}\right\rangle_{I}\right| .
$$

Next, we consider the three subintervals given by (4.6): on the first subinterval we have

$$
\begin{aligned}
\left|\varepsilon_{2}\left\langle b\left(u-\pi_{2 q-1, q} u\right), \xi^{\prime}\right\rangle_{I_{1}}\right| & \leq C \varepsilon_{2}\|b\|_{\infty, I_{1}}\left|\left\langle u-\pi_{2 q-1, q} u, \xi^{\prime}\right\rangle_{I_{1}}\right| \\
& \leq C \varepsilon_{2}\left\|u-\pi_{2 q-1, q} u\right\|_{0, I_{1}}\left\|\xi^{\prime}\right\|_{0, I_{1}} \\
& \leq C \frac{\varepsilon_{2}}{\lambda q \mu_{0}^{-1}}\left\|u-\pi_{2 q-1, q} u\right\|_{0, I_{1}}\|\xi\|_{0, I_{1}},
\end{aligned}
$$

where we used an inverse inequality; see, e.g., [15, Thm. 3.91]. Thus

$$
\left|\varepsilon_{2}\left\langle b\left(u-\pi_{2 q-1, q} u\right), \xi^{\prime}\right\rangle_{I_{1}}\right| \leq C \frac{\varepsilon_{2} \mu_{0}}{\lambda q} \varepsilon_{2} e^{-\sigma_{3} q}\|\xi\|_{E, I} \leq C e^{-\beta q}\|\xi\|_{E, I} .
$$

Similarly, on the second subinterval we have

$$
\begin{aligned}
\left|\varepsilon_{2}\left\langle b\left(u-\pi_{2 q-1, q} u\right)^{\prime}, \xi\right\rangle_{I_{2}}\right| & \leq \varepsilon_{2}\|b\|_{\infty, I_{2}}\left|\left\langle u-\pi_{2 q-1, q} u, \xi^{\prime}\right\rangle_{I_{2}}\right| \\
& \leq C \varepsilon_{2}\left\|u-\pi_{2 q-1, q} u\right\|_{0, I_{2}}\|\xi\|_{0, I_{2}} \\
& \leq C e^{-\beta q}\|\xi\|_{E, I} .
\end{aligned}
$$

Finally, on the third subinterval,

$$
\begin{aligned}
\left|\varepsilon_{2}\left\langle b\left(u-\pi_{2 q-1, q} u\right)^{\prime}, \xi\right\rangle_{I_{3}}\right| & \leq \varepsilon_{2}\|b\|_{\infty, I_{3}}\left|\left\langle u-\pi_{2 q-1, q} u, \xi^{\prime}\right\rangle_{I_{3}}\right| \\
& \leq C \varepsilon_{2}\left\|u-\pi_{2 q-1, q} u\right\|_{0, I_{3}}\left\|\xi^{\prime}\right\|_{0, I_{3}} \\
& \leq C \varepsilon_{2}\left(\frac{\varepsilon_{1}}{\varepsilon_{2}}\right)^{1 / 2} \varepsilon_{1}^{-1 / 2} e^{-\beta q}\|\xi\|_{E, I} \\
& \leq C \varepsilon_{2}^{1 / 2} e^{-\beta q}\|\xi\|_{E, I} .
\end{aligned}
$$


Therefore, by (4.12), we get

$$
\left|\varepsilon_{2}\left\langle b\left(u-\pi_{2 q-1, q} u\right)^{\prime}, \xi\right\rangle_{I}\right| \leq C e^{-\beta q}\|\xi\|_{E, I}
$$

and

$$
\|\xi\|_{E, I}^{2} \leq C e^{-\beta q}\|\xi\|_{E, I}
$$

which completes the proof.

We conclude with our main result.

THEOREM 4.6. Let $u$ be the solution to (2.1), (2.2) and let $u_{N} \in S_{q}^{2 q-1}$ be its approximation, based on the knot vector of Defintion 4.2. Then there exist positive constants $C, \sigma$, independent of $\varepsilon_{1}, \varepsilon_{2}$, such that, $\forall q \in \mathbb{N}$,

$$
\left\|u-u_{N}\right\|_{E, I} \leq C e^{-\sigma q} .
$$

Proof. We begin with the triangle inequality:

$$
\left\|u-u_{N}\right\|_{E, I} \leq\left\|u-\pi_{2 q-1, q} u\right\|_{E, I}+\left\|\pi_{2 q-1, q} u-u_{N}\right\|_{E, I},
$$

where $\pi_{2 q-1, q}$ is the approximation operator of Theorem 4.4. The first term is handled by Lemma 4.4 and the second by Lemma 4.5.

5. Numerical example. We will be considering the following BVP and we refer to [8] for more numerical experiments. Find $u$ such that

$$
\begin{aligned}
-\varepsilon_{1} u^{\prime \prime}(x)+\frac{\varepsilon_{2}}{1+x^{2}} u^{\prime}(x)+e^{-x} u(x) & =1 \text { in } I=(0,1), \\
u(0)=u(1) & =0 .
\end{aligned}
$$

An exact solution is not available, so we use as a reference solution the one computed with the highest polynomial degree, denoted by $u_{R E F}$. Instead of the error in the energy norm, we will be measuring

$$
\text { Error }:=100 \times \max _{k=1, \ldots, n}\left|u_{R E F}\left(x_{k}\right)-u_{N}\left(x_{k}\right)\right| / \max _{k=1, \ldots, n}\left|u_{R E F}\left(x_{k}\right)\right|,
$$

where $\left\{x_{k}\right\}_{k=1}^{n} \in I$ are points in $(0,1)$, chosen uniformly in the layer region and outside - we use $n=400$ in each region for our computations.

Figure 5.1 shows the Error vs. the number of degrees of freedom, DOF, in a semi-log scale. The curves indicate exponential convergence and the fact that they coincide indicates robustness. The two curves that are not on top of the rest, correspond to even smaller errors and could be due to the fact that we are using a reference solution.

6. Conclusions. In this article we performed the numerical analysis of IGA for onedimensional reaction-convection-diffusion problems with two small parameters. We established that if the knot vector is chosen appropriately and depending on the singular perturbation parameters, then $p$-refinement yields robust, exponential rates of convergence, in the energy norm. We also presented one numerical example agreeing with, and even extending the theory.

This was a step towards the study of two-dimensional SPPs, especially fourth order SPPs, for which there are very few available methods for general two-dimensional domains.

Appendix A. Here we establish eqs. (2.14)-(2.16), in the case of constant coefficients, i.e., $b(x)=b>0, c(x)=c>0$. In [16], the non-constant coefficient case is considered; we 


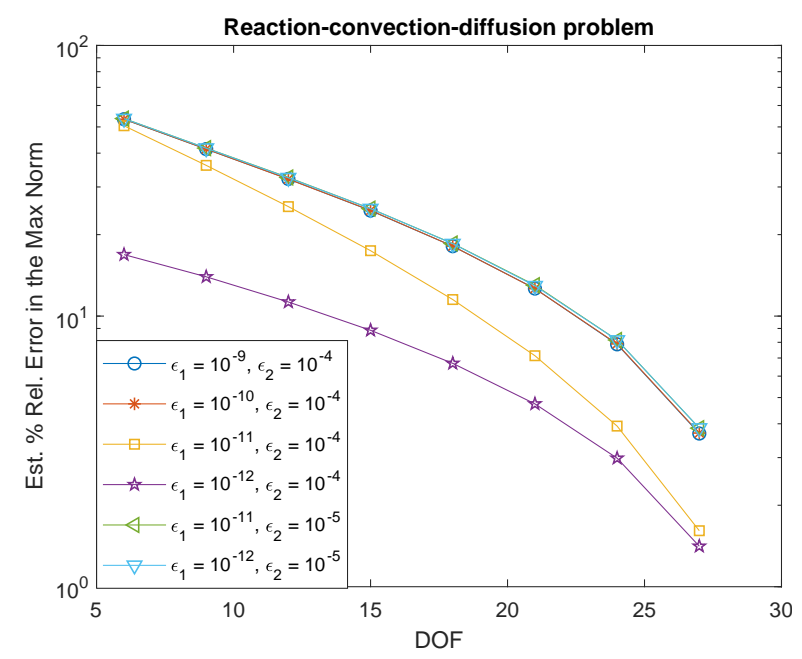

FIG. 5.1. Maximum norm convergence.

present here the much simpler case of constant coefficients in order to illustrate the procedure for obtaining such regularity estimates, for the benefit of the reader.

Recall that we are focusing on the case $\varepsilon_{1}^{2} \ll \varepsilon_{2}$, hence we anticipate a layer of width $O\left(\varepsilon_{2}\right)$ at the left endpoint and a layer of width $O\left(\varepsilon_{1} / \varepsilon_{2}\right)$ at the right endpoint. To deal with this we define the stretched variables $\tilde{x}=x / \varepsilon_{2}$ and $\hat{x}=(1-x) \varepsilon_{2} / \varepsilon_{1}$ and make the formal ansatz

$$
u \sim \sum_{i=0}^{\infty} \sum_{j=0}^{\infty} \varepsilon_{2}^{i}\left(\varepsilon_{1} / \varepsilon_{2}^{2}\right)^{j}\left(u_{i, j}(x)+\tilde{u}_{i, j}^{B L}(\tilde{x})+\hat{u}_{i, j}^{B L}(\hat{x})\right),
$$

with $u_{i, j}, \tilde{u}_{i, j}^{B L}, \hat{u}_{i, j}^{B L}$ to be determined. Substituting (A.1) into (2.1), separating the slow (i.e., $x$ ) and fast (i.e., $\tilde{x}, \hat{x}$ ) variables, and equating like powers of $\varepsilon_{1}$ and $\varepsilon_{2}$, we get

$$
\left\{\begin{array}{c}
u_{0,0}=\frac{f}{c}, \\
u_{i, 0}=-\frac{b}{c} u_{i-1,0}^{\prime}, \quad i \geq 1 \\
u_{0, j}=u_{1, j}=0, \quad j \geq 1 \\
u_{i, j}=\frac{1}{c}\left(u_{i-2, j-1}^{\prime \prime}-b u_{i-1, j}^{\prime}\right), \quad i \geq 2, j \geq 1
\end{array}\right.
$$

$$
\left\{\begin{array}{c}
b\left(\tilde{u}_{i, 0}^{B L}\right)^{\prime}+c \tilde{u}_{i, 0}^{B L}=0, \quad i \geq 0 \\
b\left(\tilde{u}_{i, j}^{B L}\right)^{\prime}+c \tilde{u}_{i, j}^{B L}=\left(\tilde{u}_{i, j-1}^{B L}\right)^{\prime \prime}, \quad i \geq 0, j \geq 1
\end{array}\right.
$$

$$
\left\{\begin{array}{c}
\left(\hat{u}_{i, 0}^{B L}\right)^{\prime \prime}+b\left(\hat{u}_{i, 0}^{B L}\right)^{\prime}=0, \quad i \geq 0, \\
\left(\hat{u}_{i, j+1}^{B L}\right)^{\prime \prime}+b\left(\hat{u}_{i, j+1}^{B L}\right)^{\prime}=c \hat{u}_{i, j}^{B L}, \quad i, j \geq 0 .
\end{array}\right.
$$

The last two equations are supplemented with the following boundary conditions (in order for (2.2) to be satisfied) for all $i, j \geq 0$ :

$$
\left\{\begin{array}{c}
\tilde{u}_{i, j}^{B L}(0)=-u_{i, j}(0), \\
\hat{u}_{i, j}^{B L}(0)=-u_{i, j}(1), \\
\lim _{\hat{x} \rightarrow-\infty} \hat{u}_{i, j}^{B L}(\hat{x})=0 .
\end{array}\right.
$$


Note that, by (A.3) and the fact that $b, c>0$, we automatically have $\lim _{\tilde{x} \rightarrow \infty} \tilde{u}_{i, j}^{B L}(\tilde{x})=0$.

Next, we would like to describe the regularity of the functions $u_{i, j}, \tilde{u}_{i, j}^{B L}, \hat{u}_{i, j}^{B L}$, defined by (A.2)-(A.5) above. We begin with $u_{i, j}$, and we have the following.

LEMMA A.1. Let $u_{i, j}$ be defined by (A.2) and assume (2.6) holds. Then there exist positive constants $C, K$ and a complex neighborhood $G$ of $\bar{I}$ such that the complex extension of $u$ (denoted again by $u$ ) satisfies

$$
\left|u_{i, j}(z)\right| \leq C \delta^{-i} K^{i} i^{i} \quad \forall z \in G_{\delta}=\{z \in G: \operatorname{dist}(z, \partial G)>\delta\} .
$$

Proof. The proof is by induction on $i$. The case $i=0$ holds trivially, so assume the result holds for $i$ and establish it for $i+1$. Let $\tau \in(0,1)$ and let $K>0$ be a constant so that $\left(\frac{2}{K^{2}}+\frac{1}{K}\right) \leq C$. We have by (A.2), the induction hypothesis with $G_{(1-\tau) \delta} \supset G_{\delta}$, and Cauchy's Integral Theorem,

$$
\begin{aligned}
\left|u_{i+1, j}(z)\right| \leq & C\left|u_{i-1, j-1}^{\prime \prime}(z)\right|+\left|u_{i, j}^{\prime}(z)\right| \\
\leq & C\left(\frac{2}{(\tau \delta)^{2}}((1-\tau) \delta)^{-i+1} K^{i-1}(i-1)^{i-1}+\frac{1}{(\tau \delta)}((1-\tau) \delta)^{-i} K^{i} i^{i}\right) \\
\leq & C \delta^{-i-1} K^{i+1}(i+1)^{i+1}\left(\frac{1}{K^{2}} \frac{1}{(i+1)^{2}} \frac{2}{\tau^{2}(1-\tau)^{i-1}}\left(\frac{i-1}{i+1}\right)^{i-1}+\right. \\
& \left.+\frac{1}{K} \frac{1}{(i+1)} \frac{1}{\tau(1-\tau)^{i}}\left(\frac{i}{i+1}\right)^{i}\right) .
\end{aligned}
$$

Choose $\tau=1 /(i+1)$. Then

$$
\left|u_{i+1, j}(z)\right| \leq C \delta^{-i-1} K^{i+1}(i+1)^{i+1}\left(\frac{2}{K^{2}}+\frac{1}{K}\right),
$$

so by the choice of $K$ the expression in brackets is bounded and this completes the proof.

LEMMA A.2. Let $u_{i, j}$ be defined by (A.2) and assume (2.6) holds. Then there exist positive constants $C, K_{1}, K_{2}$ such that

$$
\left\|u_{i, j}^{(n)}\right\|_{\infty, I} \leq C n ! K_{1}^{n} i ! K_{2}^{i} \quad \forall n \in \mathbb{N} .
$$

Proof. This follows immediately from Lemma A.1 and Cauchy's Integral Theorem for derivatives:

$$
\left\|u_{i, j}^{(n)}\right\|_{\infty, I} \leq C \frac{n !}{(n+1)^{n}} \delta^{-i} K^{i} i^{i} e^{n} \leq C n ! K_{1}^{n} i ! K_{2}^{i},
$$

with $K_{1}=e, K_{2}=K / \delta$.

The following auxiliary lemma, which is an analog of Lemma 7.3.6 in [10], will be used in the proof of Lemma A.4.

Lemma A.3. Let $\lambda, \gamma \in \mathbb{C}$ with $\operatorname{Re}(\lambda)>0, \operatorname{Re}(\gamma)>0$. Let $F$ be an entire function satisfying, for some $i, j \in \mathbb{N}_{0}$ and $C>0$,

$$
|F(z)| \leq C \gamma^{i+j} e^{-\operatorname{Re}(\lambda z)}(i+j+|z|)^{i+j} \quad \forall z \in \mathbb{C} .
$$

Let $\alpha \in \mathbb{C}$ and let $v:(0, \infty) \rightarrow \mathbb{C}$, be the solution of the problem

$$
v^{\prime}+\lambda v=F \text { on }(0, \infty), v(0)=\alpha \text {. }
$$


Then $v$ can be extended to an entire function (denoted again by $v$ ), which satisfies

$$
|v(z)| \leq C\left(\gamma^{i+j} \frac{(i+j+|z|)^{i+j+1}}{(i+j+1)}+|\alpha|\right) e^{-\operatorname{Re}(\lambda z)} \quad \forall z \in \mathbb{C} .
$$

Proof. Using an integrating factor, we find

$$
v(z)=e^{-\operatorname{Re}(\lambda z)}\left(\alpha+\int_{0}^{z} e^{\operatorname{Re}(\lambda s)} F(s) d s\right),
$$

from which we have

$$
\begin{aligned}
|v(z)| & \leq e^{-\operatorname{Re}(\lambda z)}\left(|\alpha|+\int_{0}^{|z|}\left|e^{\operatorname{Re}(\lambda s)} F(s)\right| d s\right) \\
& \leq C e^{-\operatorname{Re}(\lambda z)}\left(|\alpha|+\gamma^{i+j} \int_{0}^{|z|}\left|(i+j+|s|)^{i+j}\right| d s\right),
\end{aligned}
$$

where we used the assumption on $F$. The result follows.

LEMMA A.4. The functions $\tilde{u}_{i, j}^{B L}$, which satisfy (A.3), are entire and there exist positive constants $C, \tilde{K}, \tilde{\gamma}$, depending only on the data, such that

$$
\left|\left(\tilde{u}_{i, j}^{B L}\right)(z)\right| \leq C \tilde{K} \tilde{\gamma}^{i+j}(i+j+|z|)^{i+j} e^{-\beta \operatorname{Re}(z)} \quad \forall z \in \mathbb{C} .
$$

Proof. The proof is by induction. We first note that from (A.3), we may calculate

$$
\tilde{u}_{i, 0}^{B L}(z)=-u_{i, 0}(0) e^{-\frac{c}{b} z}, i \geq 0 .
$$

Thus, using Lemma A.2 to bound the term $\left|u_{i, 0}(0)\right|$, we get

$$
\left|\tilde{u}_{i, 0}^{B L}(z)\right| \leq C i ! K_{2}^{i} e^{-\left|\frac{c}{b} z\right|} \leq C \tilde{\gamma}^{i}(i+|z|)^{i} e^{-\beta R e(z)},
$$

where $\tilde{\gamma}=K_{2}, \beta=c / b$, hence the result holds for $j=0$ and for all $i \geq 0$. We then proceed by induction on $j$. We assume (A.6) holds for $j \geq 1$ (and for all $i \geq 0$ ) and show it for $j+1$. We note that, by (A.3), $\tilde{u}_{i, j+1}^{B L}$ satisfies, $\forall i, j \geq 0$,

$$
\left(\tilde{u}_{i, j+1}^{B L}\right)^{\prime}+\frac{c}{b} \tilde{u}_{i, j+1}^{B L}=\frac{1}{b}\left(\tilde{u}_{i, j}^{B L}\right)^{\prime \prime} \quad, \quad \tilde{u}_{i, j+1}^{B L}(0)=-u_{i, j+1}(0) .
$$

By the induction hypothesis and Cauchy's Integral Theorem for Derivatives (we take as the contour the unit circle centered at $z$ ), we get

$$
\left|\left(\tilde{u}_{i, j}^{B L}\right)^{\prime \prime}(z)\right| \leq C \tilde{\gamma}^{i+j}(i+j+|z|)^{i+j} e^{-\beta \operatorname{Re}(z)} .
$$

Lemma A.3 is then applicable (with $\lambda=\frac{c}{b}, \gamma=\tilde{\gamma}, F=\frac{1}{b}\left(\tilde{u}_{i, j}^{B L}\right)^{\prime \prime}$ and $\alpha=-u_{i, j+1}(0)$ ) and with the aid of Lemma A.2 (to bound $|\alpha|$ ) we obtain

$$
\begin{aligned}
\left|\left(\tilde{u}_{i, j+1}^{B L}\right)(z)\right| \leq & C\left(\tilde{\gamma}^{i+j} \frac{(i+j+1+|z|)^{i+j+1}}{(i+j+1)}+i ! K_{2}^{i}\right) e^{-\operatorname{Re}(\lambda z)} \\
\leq & C \tilde{\gamma}^{i+j}(i+j+1+|z|)^{i+j+1} \times \\
& \left(\frac{1}{(i+j+1)}+\frac{i ! K_{2}^{i}}{\tilde{\gamma}^{i}(i+j+1+|z|)^{i+j+1}}\right) e^{-\operatorname{Re}(\lambda z)} \\
\leq & C \frac{\tilde{\gamma}^{i}}{(i+j+1) !}(i+j+1+|z|)^{i+j+1} e^{-\operatorname{Re}(\lambda z)}
\end{aligned}
$$


where we used the fact that $\tilde{\gamma}=K_{2}$. Hence, the induction is complete and this concludes the proof.

COROLlARY A.5. The functions $\tilde{u}_{i, j}^{B L}$, which satisfy (A.3), are entire and there exist positive constants $C, \tilde{K}, K_{1}, \tilde{\gamma}$, depending only on the data, such that, $\forall n \in \mathbb{N}_{0}$ and $\tilde{x} \geq 0$, there holds

$$
\left|\left(\tilde{u}_{i, j}^{B L}\right)^{(n)}(\tilde{x})\right| \leq C K_{1}^{n} \tilde{K} \tilde{\gamma}^{i+j}(i+j)^{i+j} e^{-\beta \tilde{x}} .
$$

Proof. Cauchy's Integral Theorem for Derivatives gives

$$
\begin{aligned}
\left|\left(\tilde{u}_{i, j}^{B L}\right)^{(n)}(\tilde{x})\right| & \leq C e^{-\beta \tilde{x}} \frac{n !}{(n+1)^{n}} \tilde{\gamma}^{i+j}(i+j+\tilde{x})^{i+j} e^{n} \\
& \leq C e^{-\beta \tilde{x}} \frac{n !}{(n+1)^{n}} \tilde{\gamma}^{i+j}(i+j+n)^{i+j} e^{n} .
\end{aligned}
$$

The proof is completed by observing that

$$
(i+j+n)^{i+j} \leq(i+j)^{i+j}(1+n /(i+j))^{i+j} \leq(i+j)^{i+j} e^{n} .
$$

REMARK A.6. An analogous result may be proven for the functions $\hat{u}_{i, j}^{B L}$, which satisfy (A.4), (A.5):

$$
\left|\left(\hat{u}_{i, j}^{B L}\right)^{(n)}(\hat{x})\right| \leq C \hat{K}^{n} \hat{\gamma}^{i+j}(i+j)^{i+j} e^{-\beta \hat{x}} \quad \forall i, j \geq 0 .
$$

Indeed, from (A.4) we find

$$
\begin{gathered}
\hat{u}_{i, 0}^{B L}(\hat{x})=\hat{u}_{i, 0}^{B L}(1) e^{-b \hat{x}} \\
\hat{u}_{i, 1}^{B L}(\hat{x})=\hat{u}_{i, 1}^{B L}(1) e^{-b \hat{x}}+\int_{1}^{\hat{x}} c \hat{u}_{i, 0}^{B L}(s)\left(1-e^{b(s-\hat{x})}\right) d s
\end{gathered}
$$

and an inductive argument, like in the proof of Lemma A.4, can be used to establish (A.8).

Next, we define, for some $M \in \mathbb{Z}$,

$$
\begin{aligned}
u_{M}(x) & =\sum_{i=0}^{M} \sum_{j=0}^{M} \varepsilon_{2}^{i}\left(\varepsilon_{1} / \varepsilon_{2}^{2}\right)^{j} u_{i, j}(x), \\
\tilde{u}_{M}^{B L}(\tilde{x}) & =\sum_{i=0}^{M} \sum_{j=0}^{M} \varepsilon_{2}^{i}\left(\varepsilon_{1} / \varepsilon_{2}^{2}\right)^{j} \tilde{u}_{i, j}^{B L}(\tilde{x}), \\
\hat{u}_{M}^{B L}(\hat{x}) & =\sum_{i=0}^{M} \sum_{j=0}^{M} \varepsilon_{2}^{i}\left(\varepsilon_{1} / \varepsilon_{2}^{2}\right)^{j} \hat{u}_{i, j}^{B L}(\hat{x}), \\
r_{M} & =u-\left(u_{M}+\tilde{u}_{M}^{B L}+\hat{u}_{M}^{B L}\right),
\end{aligned}
$$

and we have the following decomposition

$$
u=u_{M}+\tilde{u}_{M}^{B L}+\hat{u}_{M}^{B L}+r_{M} .
$$

THEOREM A.7. Assume that (2.6), (2.3) hold. Then there exist positive constants $C, K_{1}, K_{2}, \tilde{K}, \hat{K}, \tilde{\gamma}, \hat{\gamma}, \delta$, independent of $\varepsilon_{1}, \varepsilon_{2}$, such that the solution $u$ of (2.1), (2.2) can be decomposed as in (A.12), with

$$
\left\|u_{M}^{(n)}\right\|_{\infty, I} \leq C n ! K_{1}^{n} \quad \forall n \in \mathbb{N}_{0},
$$




$$
\left|\left(\tilde{u}_{M}^{B L}\right)^{(n)}(x)\right| \leq C \tilde{K}^{n} \varepsilon_{2}^{-n} e^{-\operatorname{dist}(x, \partial I) / \varepsilon_{2}} \quad \forall n \in \mathbb{N}_{0},
$$

$$
\left|\left(\hat{u}_{M}^{B L}\right)^{(n)}(x)\right| \leq C \hat{K}_{2}^{n}\left(\frac{\varepsilon_{1}}{\varepsilon_{2}}\right)^{-n} e^{-\operatorname{dist}(x, \partial I) \varepsilon_{2} / \varepsilon_{1}} \quad \forall n \in \mathbb{N}_{0},
$$

provided $\varepsilon_{2} e M \max \left\{K_{2}, \tilde{\gamma}, \hat{\gamma}\right\}<1$ and $\frac{\varepsilon_{1}}{\varepsilon_{2}^{2}} e M \max \{\tilde{\gamma}, \hat{\gamma}\}<1$.

Proof. We first show (A.13): from (A.9) and Lemma A.2 we have

$$
\begin{aligned}
\left\|u_{M}^{(n)}\right\|_{\infty, I} & \leq \sum_{i=0}^{M} \sum_{j=0}^{M} \varepsilon_{2}^{i}\left(\varepsilon_{1} / \varepsilon_{2}^{2}\right)^{j}\left\|u_{i, j}^{(n)}\right\|_{\infty, I} \leq C \sum_{i=0}^{M} \sum_{j=0}^{M} \varepsilon_{2}^{i}\left(\varepsilon_{1} / \varepsilon_{2}^{2}\right)^{j} n ! K_{1}^{n} i ! K_{2}^{i} \\
& \leq C n ! K_{1}^{n}\left(\sum_{i=0}^{M} \varepsilon_{2}^{i} i^{i} K_{2}^{i}\right)\left(\sum_{j=0}^{M}\left(\varepsilon_{1} / \varepsilon_{2}^{2}\right)^{j}\right) \\
& \leq C n ! K_{1}^{n}\left(\sum_{i=0}^{\infty}\left(\varepsilon_{2} M K_{2}\right)^{i}\right)\left(\sum_{j=0}^{\infty}\left(\varepsilon_{1} / \varepsilon_{2}^{2}\right)^{j}\right) \\
& \leq C n ! K_{1}^{n}
\end{aligned}
$$

since both sums are convergent geometric series due to the assumptions $\varepsilon_{2} M K_{2}<1$ and $\varepsilon_{1} / \varepsilon_{2}^{2}<1$.

Next, we show (A.14): by (A.10) and Lemma A.4, we have

$$
\begin{aligned}
\left|\left(\tilde{u}_{M}^{B L}\right)^{(n)}(\tilde{x})\right| & \leq \sum_{i=0}^{M} \sum_{j=0}^{M} \varepsilon_{2}^{i}\left(\varepsilon_{1} / \varepsilon_{2}^{2}\right)^{j}\left|\left(\tilde{u}_{i, j}^{B L}\right)^{(n)}(\tilde{x})\right| \\
& \leq C \sum_{i=0}^{M} \sum_{j=0}^{M} \varepsilon_{2}^{i}\left(\varepsilon_{1} / \varepsilon_{2}^{2}\right)^{j} \tilde{K}^{n} \tilde{\gamma}^{i}(i+j)^{i+j} e^{-|\beta z|} .
\end{aligned}
$$

Now, $(i+j)^{i+j} \leq e^{i} i^{i} e^{j} j^{j}$ (cf. (A.7)) hence we get

$$
\begin{aligned}
\left|\left(\tilde{u}_{M}^{B L}\right)^{(n)}(\tilde{x})\right| & \leq C \tilde{K}^{n} e^{-\beta \tilde{x}}\left(\sum_{i=0}^{M} \tilde{\gamma}^{i} e^{i} i^{i} \varepsilon_{2}^{i}\right)\left(\sum_{j=0}^{M}\left(\varepsilon_{1} / \varepsilon_{2}^{2}\right)^{j} e^{j} j^{j}\right) \\
& \leq C \tilde{K}^{n} e^{-\beta \tilde{x}}\left(\sum_{i=0}^{\infty}\left(\tilde{\gamma} e M \varepsilon_{2}\right)^{i}\right)\left(\sum_{j=0}^{\infty}\left(\frac{\varepsilon_{1}}{\varepsilon_{2}^{2}} e M\right)^{j}\right) \\
& \leq C \tilde{K}^{n} e^{-\beta \tilde{x}}
\end{aligned}
$$

since both sums are convergent geometric series, due to the assumptions $\tilde{\gamma} e M \varepsilon_{2}<1$, $\frac{\varepsilon_{1}}{\varepsilon_{2}^{2}} e M<1$. The result follows. 
Similarly, we show (A.15): by (A.11) and (A.8),

$$
\begin{aligned}
\left|\left(\hat{u}_{M}^{B L}\right)^{(n)}(\hat{x})\right| & \leq \sum_{i=0}^{M} \sum_{j=0}^{M} \varepsilon_{2}^{i}\left(\varepsilon_{1} / \varepsilon_{2}^{2}\right)^{j}\left|\left(\hat{u}_{i, j}^{B L}\right)^{(n)}(\hat{x})\right| \\
& \leq C \sum_{i=0}^{M} \sum_{j=0}^{M} \varepsilon_{2}^{i}\left(\varepsilon_{1} / \varepsilon_{2}^{2}\right)^{j} \hat{K}^{n} \hat{\gamma}^{i}(i+j)^{i+j} e^{-\beta \hat{x}} \\
& \leq C \hat{K}^{n} e^{-\beta \hat{x}}\left(\sum_{i=0}^{\infty}\left(\hat{\gamma} e M \varepsilon_{2}\right)^{i}\right)\left(\sum_{j=0}^{\infty}\left(\frac{\varepsilon_{1}}{\varepsilon_{2}^{2}} e M\right)^{j}\right) \\
& \leq C \hat{K}^{n} e^{-\beta \hat{x}} .
\end{aligned}
$$

It remains to show (A.16). To this end, note that

$$
\begin{aligned}
r_{M}(0) & =u(0)-\sum_{i=0}^{M} \sum_{j=0}^{M} \varepsilon_{2}^{i}\left(\varepsilon_{1} / \varepsilon_{2}^{2}\right)^{j}\left(u_{i, j}(0)+\tilde{u}_{i, j}^{B L}(0)+\hat{u}_{i, j}^{B L}\left(\varepsilon_{2} / \varepsilon_{1}\right)\right) \\
& =-\sum_{i=0}^{M} \sum_{j=0}^{M} \varepsilon_{2}^{i}\left(\varepsilon_{1} / \varepsilon_{2}^{2}\right)^{j} \hat{u}_{i, j}^{B L}\left(\varepsilon_{2} / \varepsilon_{1}\right) .
\end{aligned}
$$

By (A.8),

$$
\begin{aligned}
\left|r_{M}(0)\right| & \leq \sum_{i=0}^{M} \sum_{j=0}^{M} \varepsilon_{2}^{i}\left(\varepsilon_{1} / \varepsilon_{2}^{2}\right)^{j}\left|\hat{u}_{i, j}^{B L}\left(\varepsilon_{2} / \varepsilon_{1}\right)\right| \leq C \sum_{i=0}^{M} \sum_{j=0}^{M} \varepsilon_{2}^{i}\left(\varepsilon_{1} / \varepsilon_{2}^{2}\right)^{j} \hat{\gamma}^{i}(i+j)^{i+j} e^{-\beta \varepsilon_{2} / \varepsilon_{1}} \\
& \leq C e^{-\beta \varepsilon_{2} / \varepsilon_{1}}\left(\sum_{i=0}^{\infty}\left(\hat{\gamma} M e \varepsilon_{2}\right)^{i}\right)\left(\sum_{j=0}^{\infty}\left(\left(\varepsilon_{1} / \varepsilon_{2}^{2}\right) e M\right)^{j}\right) \leq C e^{-\delta \varepsilon_{2} / \varepsilon_{1}},
\end{aligned}
$$

for some positive $\delta$, independent of $\varepsilon_{1}, \varepsilon_{2}$ and bounded away from 0 . Similarly,

$$
\begin{aligned}
\left|r_{M}(1)\right| & \leq \sum_{i=0}^{M} \sum_{j=0}^{M} \varepsilon_{2}^{i}\left(\varepsilon_{1} / \varepsilon_{2}^{2}\right)^{j}\left|\tilde{u}_{i, j}^{B L}\left(1 / \varepsilon_{2}\right)\right| \leq C \sum_{i=0}^{M} \sum_{j=0}^{M} \varepsilon_{2}^{i}\left(\varepsilon_{1} / \varepsilon_{2}^{2}\right)^{j} \tilde{\gamma}^{i}(i+j)^{i+j} e^{-\beta / \varepsilon_{2}} \\
& \leq C e^{-\beta / \varepsilon_{2}}\left(\sum_{i=0}^{\infty}\left(\tilde{\gamma} e \varepsilon_{2} M\right)^{i}\right)\left(\sum_{j=0}^{\infty}\left(\left(\varepsilon_{1} / \varepsilon_{2}^{2}\right) M\right)^{j}\right) \leq C e^{-\delta / \varepsilon_{2}} .
\end{aligned}
$$

Combining the two results, we have

$$
\left\|r_{M}\right\|_{\infty, \partial I} \leq C \max \left\{e^{-\delta \varepsilon_{2} / \varepsilon_{1}}, e^{-\delta / \varepsilon_{2}}\right\} .
$$

Now, let $L:=-\varepsilon_{1} \frac{d^{2}}{d x^{2}}+\varepsilon_{2} b \frac{d}{d x}+c$ Id, with Id the identity operator, and consider

$$
L\left(u-u_{M}\right)=f(x)-\sum_{i=0}^{M} \sum_{j=0}^{M} \varepsilon_{2}^{i}\left(\varepsilon_{1} / \varepsilon_{2}^{2}\right)^{j} L u_{i, j}(x),
$$

with $u_{i, j}$ satisfying (A.2). After some calculations, we find

$$
L\left(u-u_{M}\right)=-\varepsilon_{2}^{M+1} \sum_{j=1}^{M}\left(\frac{\varepsilon_{1}}{\varepsilon_{2}^{2}}\right)^{j} b u_{M, j}^{\prime},
$$


hence

$$
\begin{aligned}
\left\|L\left(u-u_{M}\right)\right\|_{\infty, I} & \leq \varepsilon_{2}^{M+1} \sum_{j=1}^{M}\left(\frac{\varepsilon_{1}}{\varepsilon_{2}^{2}}\right)^{j}\|b\|_{\infty, I}\left\|u_{M, j}^{\prime}\right\|_{\infty, I} \\
& \leq C \varepsilon_{2}^{M+1} \sum_{j=1}^{M}\left(\frac{\varepsilon_{1}}{\varepsilon_{2}^{2}}\right)^{j}\left\|u_{M, j}^{\prime}\right\|_{\infty, I} .
\end{aligned}
$$

Using Lemma A.2, we further obtain

$$
\left\|L\left(u-u_{M}\right)\right\|_{\infty, I} \leq C \varepsilon_{2}^{M+1} M ! K_{2}^{M} \sum_{j=1}^{M}\left(\frac{\varepsilon_{1}}{\varepsilon_{2}^{2}}\right)^{j} \leq C \varepsilon_{2}\left(\varepsilon_{2} M K_{2}\right)^{M}
$$

since the finite sum can be bounded by a converging geometric series.

We also consider the operator $L$ in the stretched variable $\tilde{x}$ :

$$
\tilde{L}=-\varepsilon_{1} \varepsilon_{2}^{-2} \frac{d^{2}}{d \tilde{x}^{2}}+b \frac{d}{d \tilde{x}}+c \mathrm{Id}
$$

and we find, after some calculations,

$$
\begin{aligned}
\tilde{L} \tilde{u}_{M}^{B L} & =\sum_{i=0}^{M} \sum_{j=0}^{M} \varepsilon_{2}^{i}\left(\varepsilon_{1} / \varepsilon_{2}^{2}\right)^{j} \tilde{L}_{u_{i, j}^{B L}} \\
& =\sum_{i=0}^{M} \sum_{j=0}^{M} \varepsilon_{2}^{i}\left(\varepsilon_{1} / \varepsilon_{2}^{2}\right)^{j}\left(-\varepsilon_{1} \varepsilon_{2}^{-2}\left(\tilde{u}_{i, j}^{B L}\right)^{\prime \prime}+b\left(\tilde{u}_{i, j}^{B L}\right)^{\prime}+c \tilde{u}_{i, j}^{B L}\right) \\
& =\left(\frac{\varepsilon_{1}}{\varepsilon_{2}^{2}}\right)^{M+1} \sum_{i=0}^{M} \varepsilon_{2}^{i}\left(\tilde{u}_{i, M}^{B L}\right)^{\prime \prime}
\end{aligned}
$$

where (A.3) was used. Hence, using (A.6), we have

$$
\begin{aligned}
\left\|\tilde{L} \tilde{u}_{M}^{B L}\right\|_{\infty, I} & \leq\left(\frac{\varepsilon_{1}}{\varepsilon_{2}^{2}}\right)^{M+1} \sum_{i=0}^{M} \varepsilon_{2}^{i}\left\|\left(\tilde{u}_{i, M}^{B L}\right)^{\prime \prime}\right\|_{\infty, I} \leq C\left(\frac{\varepsilon_{1}}{\varepsilon_{2}^{2}}\right)^{M+1} \sum_{i=0}^{M} \varepsilon_{2}^{i} \tilde{\gamma}^{i}(i+M)^{i+M} \\
& \leq C\left(\frac{\varepsilon_{1}}{\varepsilon_{2}^{2}}\right)^{M+1} \sum_{i=0}^{M} \varepsilon_{2}^{i} \tilde{\gamma}^{i} e^{i} i^{i} e^{M} M^{M} \leq C\left(\frac{\varepsilon_{1}}{\varepsilon_{2}^{2}} e M\right)^{M+1} \sum_{i=0}^{M}\left(\varepsilon_{2} \tilde{\gamma} e M\right)^{i} \\
& \leq C\left(\frac{\varepsilon_{1}}{\varepsilon_{2}^{2}} e M\right)^{M+1} .
\end{aligned}
$$

Similarly, in the stretched variable $\hat{x}$ we have

$$
\hat{L}=-\varepsilon_{1}\left(\frac{\varepsilon_{2}}{\varepsilon_{1}}\right)^{2} \frac{d^{2}}{d \hat{x}^{2}}-b \varepsilon_{2} \frac{\varepsilon_{2}}{\varepsilon_{1}} \frac{d}{d \hat{x}}+c \text { Id }
$$


and, with the help of (A.4),

$$
\begin{aligned}
\hat{L} \hat{u}_{M}^{B L} & =\sum_{i=0}^{M} \sum_{j=0}^{M} \varepsilon_{2}^{i}\left(\varepsilon_{1} / \varepsilon_{2}^{2}\right)^{j} \hat{L} \hat{u}_{i, j}^{B L} \\
& =\sum_{i=0}^{M} \sum_{j=0}^{M} \varepsilon_{2}^{i}\left(\varepsilon_{1} / \varepsilon_{2}^{2}\right)^{j}\left(-\frac{\varepsilon_{2}^{2}}{\varepsilon_{1}}\left(\hat{u}_{i, j}^{B L}\right)^{\prime \prime}-b \frac{\varepsilon_{2}^{2}}{\varepsilon_{1}}\left(\hat{u}_{i, j}^{B L}\right)^{\prime}+c \hat{u}_{i, j}^{B L}\right) \\
& =\left(\frac{\varepsilon_{1}}{\varepsilon_{2}^{2}}\right)^{M} \sum_{i=0}^{M} \varepsilon_{2}^{i}\left(\tilde{u}_{i, M}^{B L}\right)^{\prime \prime},
\end{aligned}
$$

thus, by following the exact same steps as above,

$$
\begin{aligned}
\left\|\hat{L} \hat{u}_{M}^{B L}\right\|_{\infty, I} & \leq\left(\frac{\varepsilon_{1}}{\varepsilon_{2}^{2}}\right)^{M} \sum_{i=0}^{M} \varepsilon_{2}^{i}\left\|\left(\hat{u}_{i, M}^{B L}\right)^{\prime \prime}\right\|_{\infty, I} \leq C\left(\frac{\varepsilon_{1}}{\varepsilon_{2}^{2}}\right)^{M} \sum_{i=0}^{M} \varepsilon_{2}^{i} \hat{\gamma}^{i}(i+M)^{i+M} \\
& \leq C\left(\frac{\varepsilon_{1}}{\varepsilon_{2}^{2}} e M\right)^{M}
\end{aligned}
$$

Therefore,

$$
\begin{aligned}
\left\|L r_{M}\right\|_{\infty, I} & =\left\|L\left(u-u_{M}-\tilde{u}_{M}^{B L}-\hat{u}_{M}^{B L}\right)\right\|_{\infty, I} \\
& \leq\left\|L\left(u-u_{M}\right)\right\|_{\infty, I}+\left\|L \tilde{u}_{M}^{B L}\right\|_{\infty, I}+\left\|L \hat{u}_{M}^{B L}\right\|_{\infty, I} \\
& \leq C\left(\varepsilon_{2}\left(\varepsilon_{2} M K_{2}\right)^{M}+\left(\frac{\varepsilon_{1}}{\varepsilon_{2}^{2}} e M\right)^{M+1}\left(\frac{\varepsilon_{1}}{\varepsilon_{2}^{2}} e M\right)^{M}\right) .
\end{aligned}
$$

Under the assumptions of the theorem, we have shown that the remainder $r_{M}$ has exponentially small values at the endpoints of $I$, and $L r_{M}$ is uniformly bounded by an arbitrarily small quantity on $I$. By, e.g., stability (see [9]) we have the desired result.

\section{REFERENCES}

[1] G. E. Andrews, R. Askey, And R. Roy, Special Functions, Cambridge University Press, Cambridge, 1999.

[2] A. Buffa, G. SANGALLI, AND C. Schwab, Exponential convergence of the hp version of isogeometric analysis in $1 D$, in Spectral and High Order Methods for Partial DifferentialEequations-ICOSAHOM 2012, M. Azaiez, H. El Fekih, J. S. Hesthaven, eds, Lect. Notes Comput. Sci. Eng., 95, Springer, Cham (2014), pp. 191-203.

[3] J. A. Cottrell, T. R. Hughes, And Y. BAsilevs, Isogeometric Analysis: Toward Integration of CAD and FEA, Wiley, Chichester, 2009.

[4] L. Beirão Da Veiga, A. Buffa, J. Rivas, ANd G. SANGALLI, Some estimates for $h$-p- $k$ refinement in isogeometric analysis, Numer. Math., 118 (2011), pp. 271-305.

[5] M. G. Cox, The numerical evaluation of B-splines, National Physics Laboratory Report DNAC 4, Teddington, 1971.

[6] C. De Boor, On calculation with B-splines, J. Approx. Theory, 6 (1972), pp. 50-62.

[7] T. R. Hughes, J. A. CotTrell, AND Y. BAsILEvs, Isogeometric analysis: CAD, finite elements, NURBS, exact geometry and mesh refinement, Comput. Meth. Appl. Mech. Eng., 194 (2005), pp. 4125-4195.

[8] K. LIOTATI AND C. XENOPHONTOS, Isogeometric analysis for singularly perturbed problems in 1-D: a numerical study, Preprint on arXiv, 2018, http://arxiv.org/abs/1901.01949. To appear in the Proceedings of BAIL 2018.

[9] T. Linss, Layer-Adapted Meshes for Reaction-Convection-Diffusion Problems, Springer, Berlin, 2010.

[10] J. M. MELENK, On the robust exponential convergence of hp finite element methods for problems with boundary layers, IMA J. Numer. Anal., 17 (1997), pp. 577-601. 


\section{ETNA}

Kent State University and

Johann Radon Institute (RICAM)

IGA FOR SPP

[11] J. M. MELEnK And C. SCHWAB, An hp finite element method for convection-diffusion problems in onedimension, IMA J. Numer. Anal., 19 (1999), pp. 425-453.

[12] J. M. MELENK AND C. XENOPHontos, Robust exponential convergence of hp-FEM in balanced norms for singularly perturbed reaction-diffusion equations, Calcolo, 53 (2016), pp. 105-132.

[13] H.-G. Roos, M. STynes, And L. ToBis KA, Robust Numerical Methods for Singularly Perturbed Differential Equations, Springer, Berlin, 2008.

[14] H.-G. ROOS AND Z. UZELAC, The SDFEM for a convection-diffusion problem with two small parameters, Comput. Methods Appl. Math., 3 (2003), pp. 443-458.

[15] C. SchwAB, $p$ - and hp-Finite Element Methods, Oxford University Press, New York, 1998.

[16] I. SYKOPETRITOU AND C. XENOPHONTOS, Analytic regularity for a singularly perturbed reaction-convectiondiffusion boundary value problem with two small parameters, Preprint on arXiv, 2019.

http://arxiv.org/abs/1901.09397 Article to appear in the Mediterranean Journal of Mathematics.

[17] L. TEOFAnOV AND H.-G. Roos, An elliptic singularly perturbed problem with two parameters I: Solution decompostition, J. Comput. Appl. Math., 206 (2007), pp. 1802-1097. 